\title{
Partial recovery of visual function in a blind patient after optogenetic therapy
}

\author{
José-Alain Sahel ${ }^{1,2,3,4} \llbracket$, Elise Boulanger-Scemama ${ }^{3,4}$, Chloé Pagot $\mathbb{D}^{5}$, Angelo Arleo', \\ Francesco Galluppi ${ }^{6}$, Joseph N. Martel ${ }^{2}$, Simona Degli Esposti ${ }^{7}$, Alexandre Delaux ${ }^{1} 1$, \\ Jean-Baptiste de Saint Aubert ${ }^{1}$, Caroline de Montleau ${ }^{5}{ }^{5}$, Emmanuel Gutman ${ }^{5}$, Isabelle Audo ${ }^{1,3}$, \\ Jens Duebel', Serge Picaud ${ }^{1}{ }^{1}$, Deniz Dalkara ${ }^{\circledR 1}$, Laure Blouin ${ }^{6}$, Magali Taiel ${ }^{6}$ and Botond Roska $\mathbb{D}^{8,9 凶}$
}

Optogenetics may enable mutation-independent, circuit-specific restoration of neuronal function in neurological diseases. Retinitis pigmentosa is a neurodegenerative eye disease where loss of photoreceptors can lead to complete blindness. In a blind patient, we combined intraocular injection of an adeno-associated viral vector encoding ChrimsonR with light stimulation via engineered goggles. The goggles detect local changes in light intensity and project corresponding light pulses onto the retina in real time to activate optogenetically transduced retinal ganglion cells. The patient perceived, located, counted and touched different objects using the vector-treated eye alone while wearing the goggles. During visual perception, multichannel electroencephalographic recordings revealed object-related activity above the visual cortex. The patient could not visually detect any objects before injection with or without the goggles or after injection without the goggles. This is the first reported case of partial functional recovery in a neurodegenerative disease after optogenetic therapy.

1 tinitis pigmentosa $(\mathrm{RP})$ is a progressive, inherited, monogenic or rarely digenic ${ }^{1}$ blinding disease caused by mutations in more than 71 different genes (https://sph.uth.edu/retnet/ sum-dis.htm). It affects more than 2 million people worldwide. With the exception of a gene replacement therapy for one form of early-onset RP caused by mutation in the gene RPE65 (ref. ${ }^{2}$ ), there is no approved therapy for RP.

Optogenetic vision restoration ${ }^{3-5}$ is a mutation-independent approach for restoring visual function at the late stages of $\mathrm{RP}$ after vision is lost $^{6-9}$. The open-label phase $1 / 2$ a PIONEER study (ClinicalTrials.gov identifier: NCT03326336; the clinical trial protocol is provided in the Supplementary Text) was designed to evaluate the safety (primary objective) and efficacy (secondary objective) of an investigational treatment for patients with advanced nonsyndromic RP that combines injection of an optogenetic vector (GS030-Drug Product (GS030-DP)) with wearing a medical device, namely light-stimulating goggles (GS030-Medical Device (GS030-MD)). The proof of concept for GS030-DP and the GS030-DP dose used in the PIONEER clinical trial were established in nonhuman primate studies ${ }^{10,11}$.

The optogenetic vector, a serotype $2.7 \mathrm{~m} 8$ (ref. ${ }^{12}$ ) adenoassociated viral vector encoding the light-sensing channelrhodopsin protein ChrimsonR fused to the red fluorescent protein tdTomato $^{13}$, was administered by a single intravitreal injection into the worse-seeing eye to target mainly foveal retinal ganglion cells ${ }^{10}$. The fusion protein tdTomato was included to increase the expression of ChrimsonR in the cell membrane ${ }^{10}$. The peak sensitivity of ChrimsonR-tdTomato is around $590 \mathrm{~nm}$ (amber color) ${ }^{13}$. We chose
ChrimsonR, which has one of the most red-shifted action spectra among the available optogenetic sensors because amber light is safer and causes less pupil constriction ${ }^{10}$ than the blue light used to activate many other sensors. The light-stimulating goggles capture images from the visual world using a neuromorphic camera that detects changes in intensity, pixel by pixel, as distinct events ${ }^{14}$. The goggles then transform the events into monochromatic images and project them in real time as local 595-nm light pulses onto the retina (Extended Data Fig. 1).

\section{Results}

Safety of the optogenetic vector and light-stimulating goggles. In this article, we describe the partial recovery of vision in one participant of the PIONEER study. At the inclusion in the study, this 58-year-old male, who was diagnosed with RP 40 years ago, had a visual acuity limited to light perception. The worse-seeing eye was treated with $5.0 \times 10^{10}$ vector genomes of optogenetic vector. Both before and after the injection, we performed ocular examinations and assessed the anatomy of the retina based on optical coherence tomography images, color fundus photographs and fundus autofluorescence images taken on several occasions over 15 visits spanning 84 weeks according to the protocol (Extended Data Fig. 2). We monitored potential intraocular inflammation according to the international guidelines of the Standardization of Uveitis Nomenclature Working Group ${ }^{15,16}$ (further details are provided in the clinical trial protocol). In addition, we assessed vital signs at each visit and performed a general examination and electrocardiogram before and after the injection. There was no intraocular inflammation, no changes in

\footnotetext{
'Sorbonne Université, Institut National de la Santé et de la Recherche Médicale, Centre National de la Recherche Scientifique, Institut de la Vision, Paris, France. ${ }^{2}$ Department of Ophthalmology, The University of Pittsburgh School of Medicine, Pittsburgh, PA, USA. ${ }^{3}$ Institut National de la Santé et de la Recherche Médicale-Centre d'Investigation Clinique 1423, Centre Hospitalier National d'Ophtalmologie des Quinze-Vingts, Paris, France. ${ }^{4}$ Département d'Ophtalmologie, Fondation Ophtalmologique Rothschild, Paris, France. ${ }^{5}$ Streetlab, Institut de la Vision, Paris, France. ${ }^{6}$ GenSight Biologics, Paris, France. ${ }^{7}$ National Institute for Health Research Moorfields Biomedical Research Centre at Moorfields Eye Hospital and UCL Institute of Ophthalmology, London, UK. ${ }^{8}$ Institute of Molecular and Clinical Ophthalmology Basel, Basel, Switzerland. ${ }^{9}$ Department of Ophthalmology, University of Basel, Basel, Switzerland. 凶e-mail: sahelja@upmc.edu; botond.roska@iob.ch
} 
Table 1 | First test: finding the notebook or staple box

\begin{tabular}{|c|c|c|c|c|c|c|c|c|c|}
\hline \multirow[t]{2}{*}{ Stimulus } & \multicolumn{3}{|c|}{$\begin{array}{l}\text { Natural binocular: both } \\
\text { eyes open without the } \\
\text { light-stimulating goggles }\end{array}$} & \multicolumn{3}{|c|}{$\begin{array}{c}\text { Natural monocular: untreated eye } \\
\text { covered, treated eye open without } \\
\text { the light-stimulating goggles }\end{array}$} & \multicolumn{3}{|c|}{$\begin{array}{l}\text { Stimulated monocular: untreated eye } \\
\text { covered, treated eye open and stimulated } \\
\text { with the light-stimulating goggles }\end{array}$} \\
\hline & Perceive & Locate & Touch & Perceive & Locate & Touch & Perceive & Locate & Touch \\
\hline Notebook, contrast $=40 \%$ & $0 / 1$ & $0 / 1$ & $0 / 1$ & $0 / 1$ & $0 / 1$ & $0 / 1$ & $4 / 4$ & $4 / 4$ & $4 / 4$ \\
\hline Notebook, contrast $=55 \%$ & $0 / 1$ & $0 / 1$ & $0 / 1$ & $0 / 1$ & $0 / 1$ & $0 / 1$ & $4 / 5$ & $4 / 5$ & $4 / 5$ \\
\hline Notebook, Contrast $=100 \%$ & $0 / 1$ & $0 / 1$ & $0 / 1$ & $0 / 1$ & $0 / 1$ & $0 / 1$ & $4 / 4$ & $4 / 4$ & $4 / 4$ \\
\hline Staple box, contrast $=40 \%$ & $0 / 1$ & $0 / 1$ & $0 / 1$ & $0 / 1$ & $0 / 1$ & $0 / 1$ & $3 / 6$ & $3 / 6$ & $2 / 6$ \\
\hline Staple box, contrast $=55 \%$ & $0 / 1$ & $0 / 1$ & $0 / 1$ & $0 / 1$ & $0 / 1$ & $0 / 1$ & $2 / 5$ & $2 / 5$ & $1 / 5$ \\
\hline Staple box, contrast $=100 \%$ & $0 / 1$ & $0 / 1$ & $0 / 1$ & $0 / 1$ & $0 / 1$ & $0 / 1$ & $1 / 4$ & $1 / 4$ & $1 / 4$ \\
\hline
\end{tabular}

No test repetition was performed because the patient was unable to complete the task. He could not see anything and did not want to try again.

Table 2 | Second test: counting and locating tumblers

\begin{tabular}{|c|c|c|c|c|c|c|c|c|c|}
\hline \multirow[t]{2}{*}{ Stimulus } & \multicolumn{3}{|c|}{$\begin{array}{l}\text { Natural binocular: both } \\
\text { eyes open without the } \\
\text { light-stimulating goggles }\end{array}$} & \multicolumn{3}{|c|}{$\begin{array}{l}\text { Natural monocular: untreated eye } \\
\text { covered, treated eye open without } \\
\text { the light-stimulating goggles }\end{array}$} & \multicolumn{3}{|c|}{$\begin{array}{l}\text { Stimulated monocular: untreated eye } \\
\text { covered, treated eye open and stimulated } \\
\text { with the light-stimulating goggles }\end{array}$} \\
\hline & Perceive & Count & Locate & Perceive & Count & Locate & Perceive & Count & Locate \\
\hline Tumblers, contrast $=55 \%$ & $0 / 1$ & $0 / 1$ & $0 / 1$ & $0 / 1$ & $0 / 1$ & $0 / 1$ & $5 / 7$ & $5 / 7$ & $5 / 7$ \\
\hline Tumblers, contrast $=100 \%$ & $0 / 1$ & $0 / 1$ & $0 / 1$ & $0 / 1$ & $0 / 1$ & $0 / 1$ & $3 / 6$ & $3 / 6$ & $2 / 6$ \\
\hline
\end{tabular}

No test repetition was performed because the patient was unable to complete the task. He could not see anything and did not want to try again.

the anatomy of the retina and no ocular or systemic adverse events over the follow-up period (details of the findings are shown in the Supplementary Text and Extended Data Figs. 3-6). The treated eye retained light perception over the 84 weeks of testing.

We tested the light-stimulating goggles on the patient three times before vector injection (Extended Data Fig. 2). The patient did not report any change of vision or photophobia on any of these occasions. Four and a half months after the injection, we started systematic visual training using the light-stimulating goggles (Extended Data Fig. 7). Training was not started sooner because the expression of ChrimsonR-tdTomato in foveal ganglion cells stabilizes between two and six months after injection in nonhuman primates ${ }^{10}$. Seven months after the start of visual training, the patient began to report signs of visual improvement when using the goggles.

Partial recovery of visual function. We analyzed the visual improvement under three conditions with three psychophysical tests. The conditions were: (1) both eyes open without the light-stimulating goggles (natural binocular); (2) untreated eye covered, treated eye open without the goggles (natural monocular); and (3) untreated eye covered, treated eye open and stimulated with the goggles (stimulated monocular).

The first test consisted of perceiving, locating and touching a single object placed on a white table $\left(80 \times 80 \mathrm{~cm}^{2}\right.$ or $67.2^{\circ} \times 50.9^{\circ}$ visual angle, calculated based on distance from the eye) along an imaginary line $40 \mathrm{~cm}$ in front of the patient $(60-\mathrm{cm}$ distance from the eye), either $20 \mathrm{~cm}$ to the right or to the left ( $18.4^{\circ}$ visual angle relative to the middle) or in the middle (Extended Data Fig. 8). The object was either large, that is, a $12.5 \times 17.5-\mathrm{cm}^{2}$ notebook $\left(10.8^{\circ} \times 10.3^{\circ}\right)$, or small, that is, a $3 \times 5.5 \mathrm{~cm}^{2}$ staple box $\left(2.8^{\circ} \times 3.7^{\circ}\right)$, shown one by one in three different grayscale contrasts (notebook and staple box: Michelson contrast $=40,55$ and 100\%; notebook: root mean square (RMS) contrast $=0.41,0.53$ and 0.80 ; staple box: RMS contrast $=0.13$, 0.16 and 0.21 ) in random order. During the first test, the patient was unable to perceive any of the objects under natural binocular or natural monocular conditions; therefore, he did not attempt to locate or touch them (Table 1 and Supplementary Video 1). In contrast, in the stimulated monocular condition, the patient perceived the presence of, located and touched the larger object in 92\% (36 out of 39) of the trials (Table 1 and Supplementary Video 1). We performed a multivariable logistic regression analysis for success in performing the tasks, with object size (large or small), contrast (low $=40 \%$, medium $=55 \%$ or high $=100 \%$ ) and task (perceive, locate or touch) as the explanatory variables. The success rate was dependent on the size of the object, with a significantly higher rate of successful trials with the larger object than with the smaller one (36\% (16 out of 45 ); $P<0.001$, likelihood-ratio test for the effect of object size). The success rate was similar for objects at different contrasts (low $=67 \%(20$ out of 30$)$; medium $=57 \%$ (17 out of 30$)$; high $=63 \%$ (15 out of 24$)$; $P=0.29$, likelihood-ratio test for the effect of contrast), suggesting that even objects at lower contrasts generated enough retinal activity for perception. Finally, the success rate was similar for the different tasks (perceive, $64 \%$ (18 out of 28 ); locate, $64 \%$ (18 out of 28 ); touch, $57 \%$ ( 16 out of 28 ); $P=0.73$, likelihood-ratio test for the effect of task), suggesting that once the object was perceived, the patient could coordinate his motor system with the percept.

The second test included perceiving, counting and locating more than one object, that is, either two or three tumblers (Extended Data Fig. 9). The patient was asked to determine how many objects were placed on the white table and point to them without touching. Each tumbler $\left(6-\mathrm{cm}\right.$ diameter and $6-\mathrm{cm}$ height, $5.5^{\circ}$ and $8.1^{\circ}$ at $40 \mathrm{~cm}$, $4.2^{\circ}$ and $5.8^{\circ}$ at $66 \mathrm{~cm}$ ) was positioned in 1 of 6 possible positions along two imaginary lines: at $40 \mathrm{~cm}$ in front of the patient $(60-\mathrm{cm}$ distance from the eye), either $20 \mathrm{~cm}$ to the right or to the left $\left(18.4^{\circ}\right.$ relative to the middle) or in the middle; or at $66 \mathrm{~cm}$ in front of the patient (80-cm distance from the eye), either $20 \mathrm{~cm}$ to the right or to the left $\left(14^{\circ}\right)$ or in the middle. The objects were shown at three contrasts (Michelson contrast $=40,55$ and $100 \%$; RMS contrast $=0.29$, 0.33 and 0.41 ) in random order. In this test, similar to the results of the first test, the patient was unable to perceive the objects under natural binocular or natural monocular conditions; therefore, he did not attempt to count or locate them (Table 2 and Supplementary 


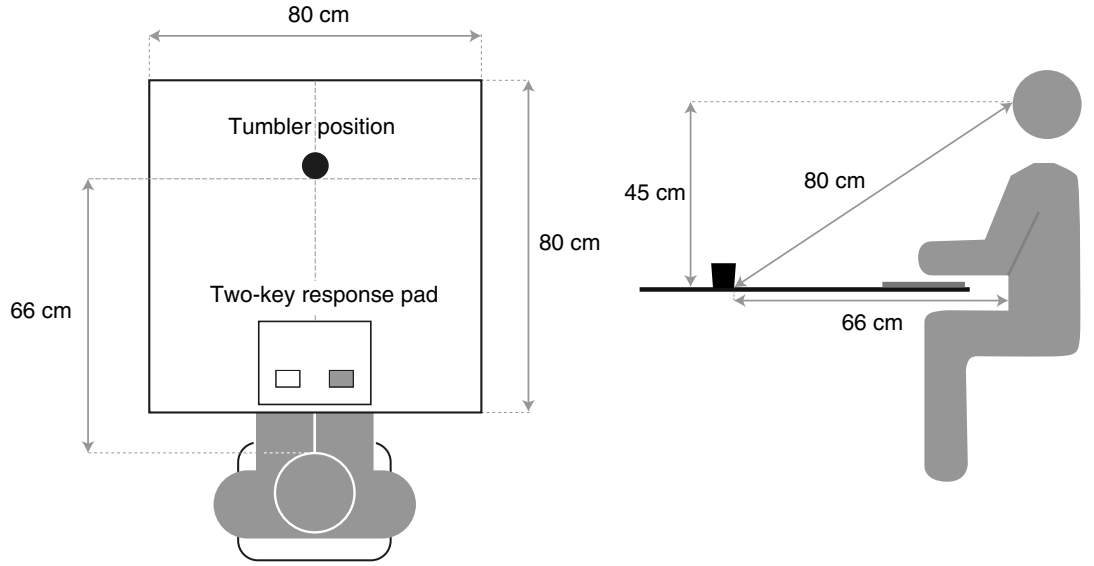

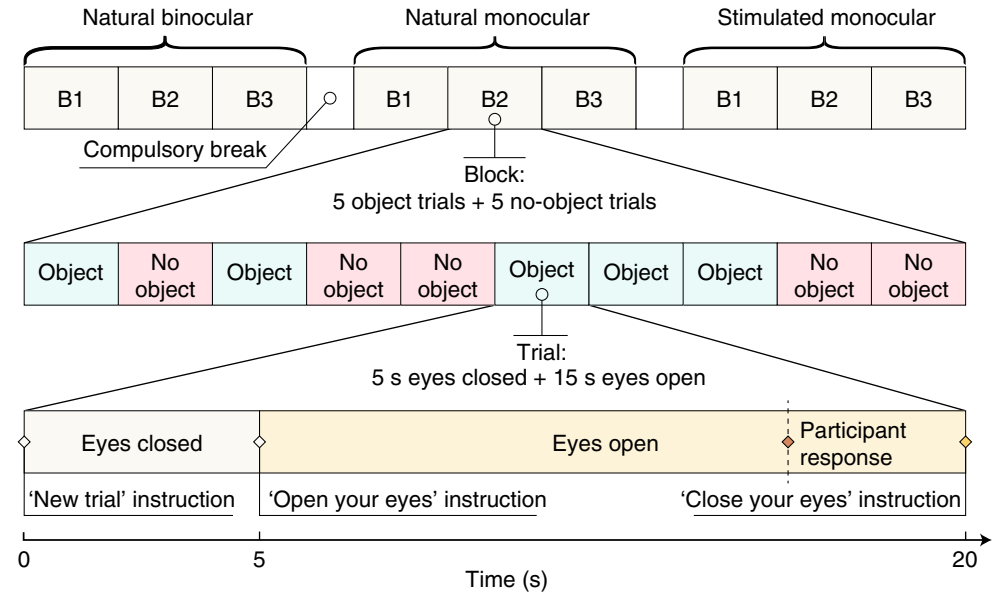

C

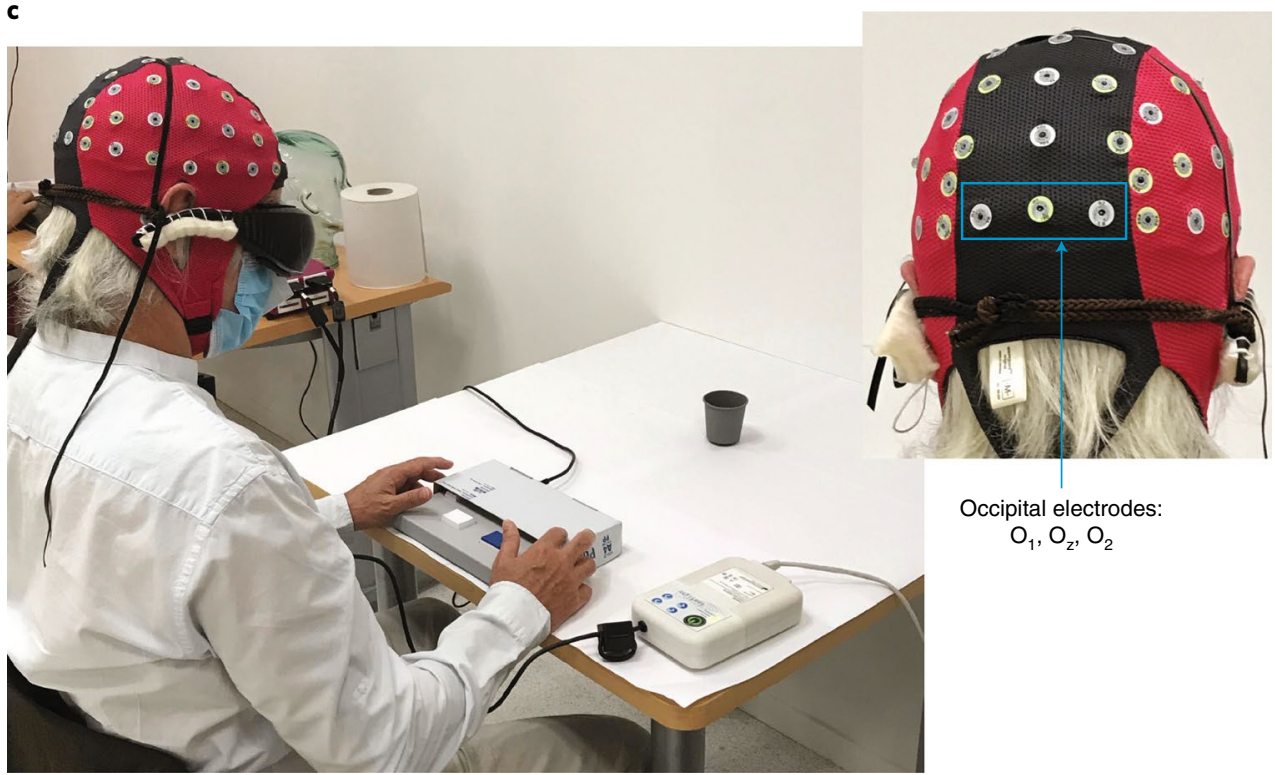

Fig. 1 | Visual task coupled with EEG recordings. a, Visual detection task. The patient had to assess the presence or absence of a tumbler $\left(6 \times 6 \mathrm{~cm}^{2}\right)$ on a white table (Michelson contrast $=55 \%$ ) through a two-key response pad. The tumbler was positioned $80 \mathrm{~cm}$ in front of the patient. $\mathbf{b}$, Experimental protocol. The entire experiment involved two sessions, with a total of 183 randomized object/no-object trials for a total duration of about 140 min. Each recording session included three conditions: natural binocular; natural monocular; and stimulated monocular. Each condition was divided into three blocks consisting of ten trials each: five object trials and five no-object trials (tumbler removed from the table). Each trial lasted 20 s. The patient was instructed to close his eyes during the first $5 \mathrm{~s}$ (while the experimenter placed/removed the tumbler on/from the table). The patient was then asked to open his eyes and was given $15 \mathrm{~s}$ to determine visually whether the tumbler was present on the table. c, Experimental setup. Behavioral responses and brain activity were simultaneously recorded during the visual test. EEG data analysis focused on the activity recorded from the occipital channels $\mathrm{O}_{1}, \mathrm{O}_{2}$ and $\mathrm{O}_{2}$. 
Table 3 | Third test: visual detection task (coupled with EEG recordings)

Trial

\begin{tabular}{llll} 
& \multicolumn{3}{c}{$\begin{array}{c}\text { eyes open without the } \\
\text { light-stimulating goggles }\end{array}$} \\
\cline { 2 - 4 } & $\begin{array}{l}\text { Answer: } \\
\text { yes object }\end{array}$ & $\begin{array}{l}\text { Answer: } \\
\text { no object }\end{array}$ & No answer \\
\hline Object trial & $3 / 30$ & $0 / 30$ & $27 / 30$ \\
No-object trial & $3 / 30$ & $1 / 30$ & $26 / 30$
\end{tabular}

Video 2). In contrast, in the stimulated monocular condition, the patient perceived the objects in $63 \%$ of the trials (12 out of 19 ). Furthermore, he correctly counted and located them in most of the trials (count, $63 \%$ (12 out of 19); locate, $58 \%$ (11 out of 19); Table 2 and Supplementary Video 2). As in the first test, the success rate was similar for objects at different contrasts (low $=67 \%$ (12 out of 18$)$; medium $=71 \%(15$ out of 21$)$; high $=44 \%(8$ out of 18$) ; P=0.20$, likelihood-ratio test for the effect of contrast).

Neural correlates of vision recovery. To investigate the link between partial vision recovery and neuronal activity, we performed a third test that combined the assessment of vision with a noninvasive brain recording technique, extracranial multichannel electroencephalography (EEG), which provides a readout of neuronal activity across the cortex (Fig. 1). This technique is more suitable than functional magnetic resonance imaging since the metallic components of the goggles are incompatible with the magnetic field generated by a magnetic resonance imaging scanner. We analyzed EEG traces in the eyes-open and eyes-closed states separately for each of the three conditions (natural binocular, natural monocular and stimulated monocular). A tumbler (6- $\mathrm{cm}$ diameter and $6-\mathrm{cm}$ height, $4.2^{\circ}$ and $5.8^{\circ}$, Michelson contrast $=55 \%$, RMS contrast $=0.33$ ) was placed or not placed on a white table in front of the patient and the patient had to assess its presence or absence. When present, the tumbler was always placed at the same position $(66 \mathrm{~cm}$ in front of the patient, $80 \mathrm{~cm}$ from the eye). We conducted a multivariable logistic regression analysis for correct assessments with condition (stimulated versus natural) and object presence (yes or no) as the explanatory variables. The rate of correct assessments was significantly higher under stimulated monocular ( $41 \%$ (26 out of 63$)$ ) than natural binocular or monocular conditions (5.8\% ( 7 out of 120 ) for both conditions; $P<0.001$, likelihood-ratio test for the effect of condition; Table 3 ).

To localize the neuronal activity with the highest information content about the visual object across the cortex, we performed a spectral analysis of the recorded signals across the 48 EEG channels in the alpha-band $(8-14 \mathrm{~Hz})$ in the eyes-open stimulated monocular condition. We found that the highest discriminant power for the object/no-object trials was located above the occipital cortex contralateral to monocular stimulation, with the most informative features corresponding to channels $\mathrm{O}_{1}$ and $\mathrm{O}_{z}$ at $14 \mathrm{~Hz}$ (Fig. 2a). We then trained a linear binary decoder with the mean alpha-power amplitudes of the occipital channels to discriminate object versus no-object trials. In the stimulated monocular condition and eyes-open state, the decoder reached a mean accuracy of $78 \%( \pm 4.8)$, which was significantly above chance level (Fig. 2b; 20-fold cross-validation, one-sided, one-sample Wilcoxon signed-rank test, $P<0.001)$. By contrast, the accuracy of decoders remained at chance level when trained under the eyes-closed state of the stimulated monocular condition, as well as under both eyes-open and eyes-closed states of the natural binocular and natural monocular conditions (Fig. 2b; eyes-closed stimulated monocular, $39 \pm 7.1 \%, P=0.93$; eyes-open natural binocular, $58 \pm 5.9 \%, P=0.08$; eyes-closed natural binocular, $60 \pm 5.7 \%, P=0.07$; eyes-open natural monocular, $52 \pm 7.1 \%$, $P=0.41$; eyes-closed natural monocular, $53 \pm 6.6 \%, P=0.23$ ). Leave-one-out cross-validation testing provided similar decoding results (eyes-open stimulated monocular, 75\%; eyes-closed stimulated monocular, $41 \%$; eyes-open natural binocular, $55 \%$; eyes-closed natural binocular, $62 \%$; eyes-open natural monocular, 55\%; eyes-closed natural monocular, 55\%). The above-chance accuracy of the decoder trained with the occipital EEG signals recorded during the eyes-open stimulated monocular condition stemmed from a modulation of the $14-\mathrm{Hz}$ alpha-power spectrum amplitude as a function of the presence/absence of the object (Fig. 2c). Object-triggered optogenetic stimulation led to a significant power decrease (that is, a desynchronization) of occipital $14-\mathrm{Hz}$ alpha oscillations (Fig. 2d; two-sided Mann-Whitney $U$-test, object versus no-object trials, $P<0.001)$.

Fig. 2 | Decoding EEG data based on power spectrum amplitude modulation of occipital alpha oscillations. a, Fisher scores for object versus no-object discrimination during the eyes-open periods in the stimulated monocular condition, calculated from power amplitude over all EEG channels for the alpha band $\left(8-14 \mathrm{~Hz}\right.$ ). Only features from the occipital channels $\mathrm{O}_{1}, \mathrm{O}_{2}$ and $\mathrm{O}_{2}$ (red) were given as input to the binary decoder. $\mathbf{b}$, Mean decoding accuracy evaluated by $k$-fold cross-validation on eyes-open and eyes-closed periods during stimulated monocular ( 63 trials), natural binocular ( 60 trials) and natural monocular ( 60 trials) conditions. The error bars indicate the s.e.m. ( $n=20$ folds). For a given condition, the data points indicate the decoding accuracy for each fold. Data points are distributed across discrete levels according to the number of randomized testing trials per fold. One-sided, one-sample Wilcoxon signed-rank test against chance level (50\%). The outcomes for each condition were: eyes-open stimulated monocular $V(19)=199$, $P=1.79 \times 10^{-4}, r=0.895$, confidence interval $(\mathrm{Cl})=0.773$, +inf; eyes-closed stimulated monocular $V(19)=66.5, P=0.933, r=0.108, \mathrm{Cl}=-0.357$, +inf; eyes-open natural binocular $V(19)=140.5, P=0.082, r=0.479, \mathrm{Cl}=0.091$, +inf; eyes-closed natural binocular $V(19)=142, P=0.073, r=0.352$, $\mathrm{Cl}=-0.051$, +inf; eyes-open natural monocular $V(19)=111.5, P=0.408, r=0.640, \mathrm{Cl}=0.282$, + inf; eyes-closed natural monocular $V(19)=124, P=0.233$, $r=0.621, \mathrm{Cl}=-0.266$, + inf. $\mathbf{c}$, Topographic representation of the mean power amplitude at $14 \mathrm{~Hz}$ over the eyes-open period in the stimulated condition averaged across trials. Top: no-object trials. Bottom: object trials. A lower power amplitude indicates a desynchronization of alpha oscillations in the visual areas, which is a signature for increased cortical excitability ${ }^{35,36}$, near-threshold stimulus perception ${ }^{36,37}$ and information transfer to downstream ventral object-selective regions ${ }^{38}$. d, Modulation of the power spectrum amplitude in the alpha band (that is, average signal in the occipital channels $\mathrm{O}_{1}$ and $\mathrm{O}_{2}$ at $14 \mathrm{~Hz}$ ) enabling the decoding of object versus no-object trials in the stimulated eyes-open condition. The horizontal red bars indicate the median, the blue boxes delimit the first and third quartiles and the error bars encompass all non-outlier data (outliers shown by red crosses). Individual data points are shown on the right. Two-sided Mann-Whitney U-test, object $(n=32)$ versus no-object $(n=31)$ trials, $W=792.5, P=4.71 \times 10^{-5}, r=0.598, \mathrm{Cl}=0.382$, 0.752. For all tests, ${ }^{\star \star} P<0.001$. Confidence intervals $(\mathrm{Cls})$ are $95 \%$. 


\section{Discussion}

In this study, we present the first evidence that injection of an optogenetic sensor-expressing gene therapy vector combined with the wearing of light-stimulating goggles can partially restore visual function in a patient with RP who had a visual acuity of only light perception.

The results of all three visual and visuomotor tests suggest that optogenetic retinal stimulation triggered by the visual scene induced a

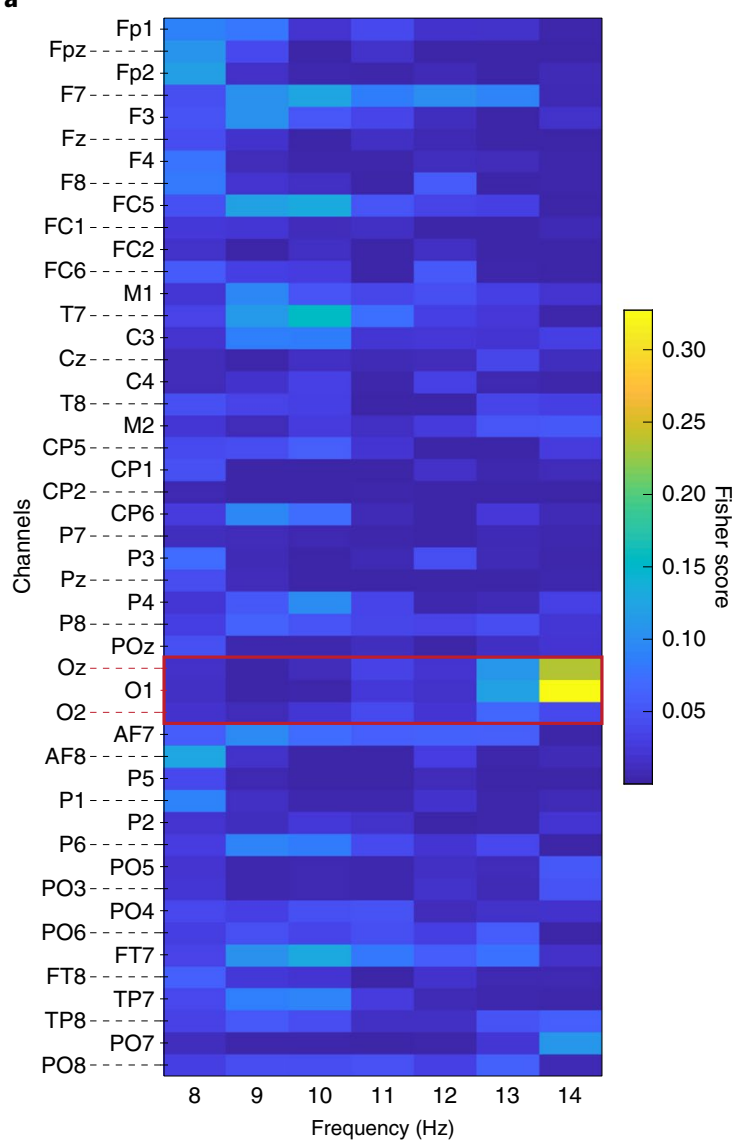

C Eyes open stimulated monocular

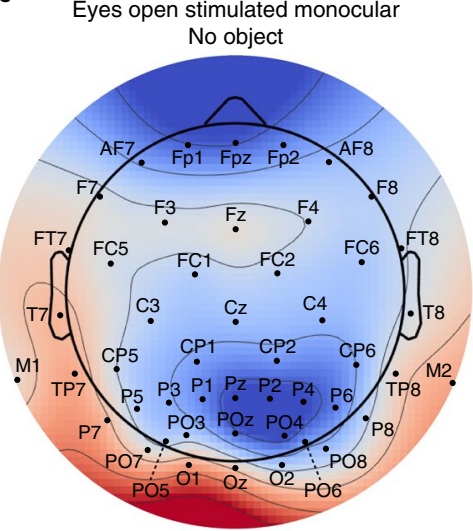

Eyes open stimulated monocular Object

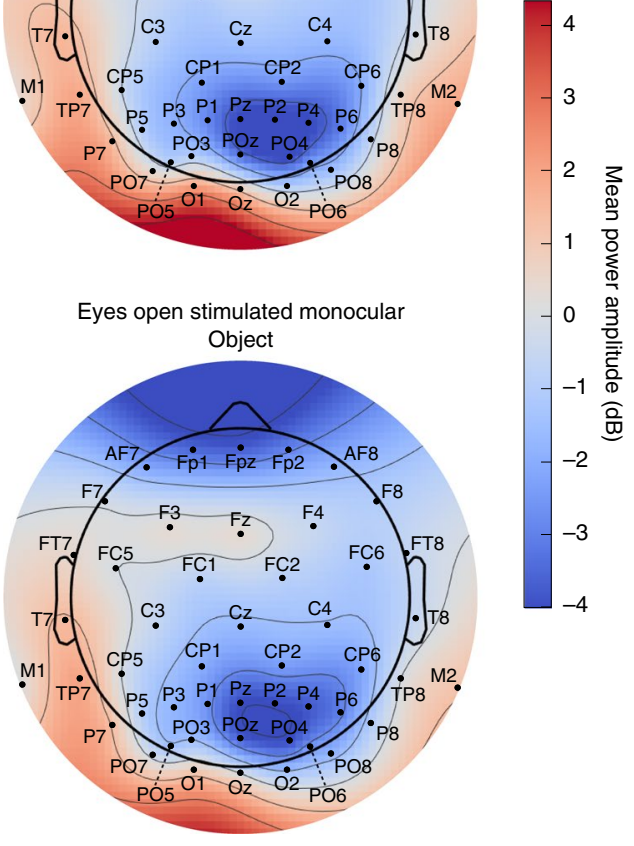

d

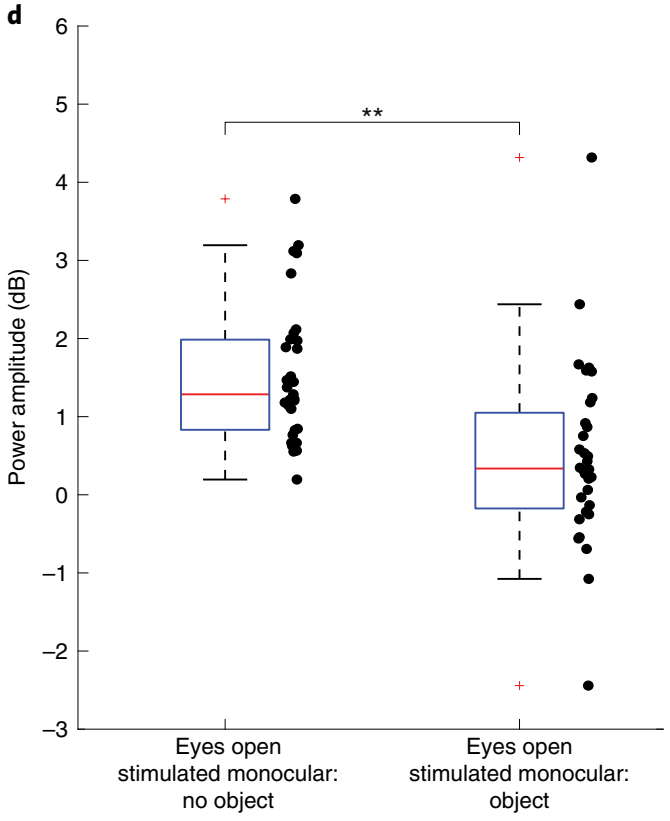

no object

object

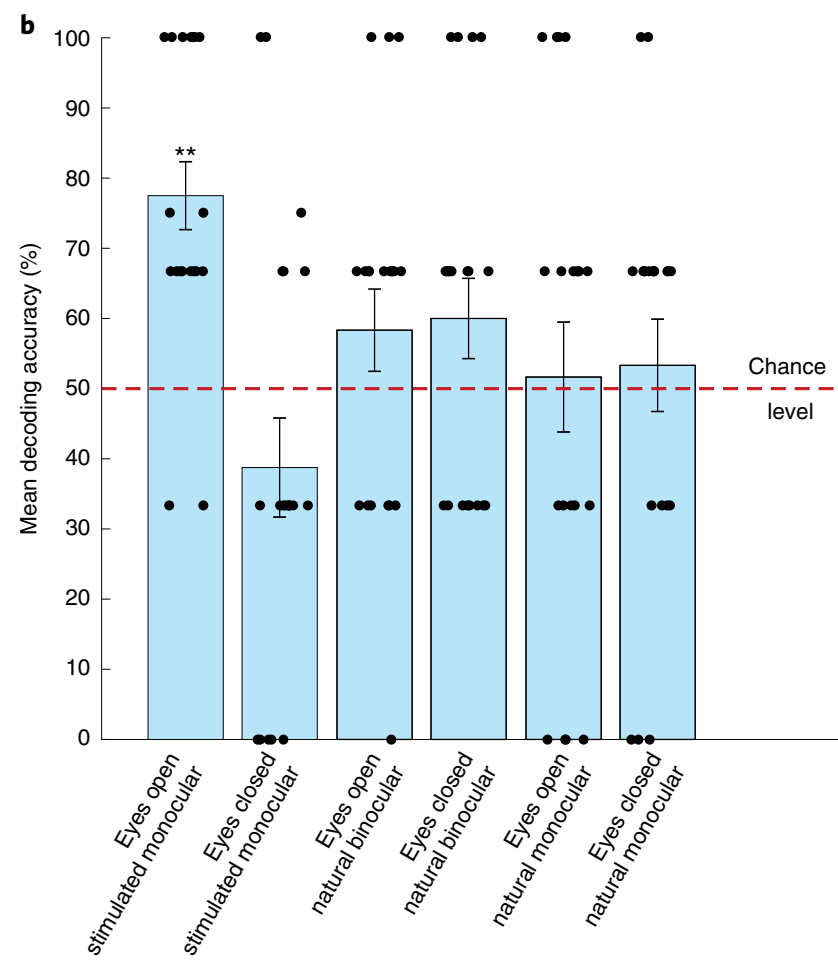


visual perception. Moreover, the visual process leading to the percept was effective enough to enable the patient to orient toward the object and perform the visuomotor task of reaching for it. The first and second tests were performed five months before the third visual test (Extended Data Fig. 2), suggesting that the gain in visual function was stable over this period.

In addition to the visual and visuomotor tests, we obtained further evidence of vision recovery in that the EEG recording of occipital cortex signals was modulated by the presence or absence of a visual object. A large body of work has coupled EEG recordings with the assessment of visual function in low-vision patients. Since the 1950s, cortical correlates of vision in visually impaired patients with RP have been investigated using EEG, through the assessment of alpha rhythm modulations ${ }^{17-21}$, as well as using visually evoked potentials $^{22-25}$. Furthermore, other characteristics of the EEG signal have been used to investigate the neural correlates in low-vision patients or patients with functional visual recovery. For instance, substantial differences to healthy individuals have been found during visual stimulation of patients with inherited retinal dystrophies using the shape of the EEG power spectral density, in particular in the occipital electrodes ${ }^{26}$. Posterior alpha and theta activity related to the visual processing of motion has also been used to identify the neural mechanisms of functional sight restoration ${ }^{27}$.

In this study, the EEG recordings suggested that retinal activity evoked by the optogenetic stimulation of the retina propagates to the primary visual cortex and modulates its activity. This cortical activity, quantified as changes in amplitude of the local EEG alpha power, conveys sufficient information to allow object versus no-object stimulations to be decoded on a single-trial basis. Our findings are consistent with the involvement of occipital alpha rhythm fluctuations in object-based visual attention ${ }^{28}$ and processing ${ }^{29}$, top-down control of visual attention (for example, to mediate forthcoming visual stimulation processing ${ }^{30-33}$ ), stimulus discrimination in object detection ${ }^{29}$ and object recognition $^{34}$. Our analysis of the EEG power spectrum shows that visual object-triggered optogenetic stimulation induces desynchronization of occipital EEG oscillations in the upper alpha range, as shown by a significant decrease in the $14-\mathrm{Hz}$ power amplitude compared to the absence of the object. Alpha-power decrease in visual areas has been associated with increased cortical excitability ${ }^{35,36}$, near-threshold stimulus perception ${ }^{36,37}$ and transfer of information to ventral object-selective regions ${ }^{38}$. Thus, occipital alpha desynchronization induced by visual object-triggered optogenetic stimulation is likely a neurophysiological confirmation of the individual's partially recovered visual perception.

In attempts to detect the presence of objects during the visual tests, the patient adopted a head-scanning strategy when using the light-stimulating goggles. There are two possible reasons for this. Either the field of optogenetic activation was too small to detect objects not aligned with the camera center or there was no spatial resolution within the area of the retina that expressed the optogenetic sensor. Based on experiments in nonhuman primates ${ }^{10}$, we estimated the region of optogenetic expression in human retina to be $8.20^{\circ}$ of visual angle $(2.5-\mathrm{mm}$ diameter retinal disc). Moreover, using the same vector and injection method reported in this article, electrophysiological experiments in nonhuman primate retinas demonstrated spatial resolution within the foveal ganglion cell array ${ }^{10}$. Therefore, we hypothesize that the patient's need to scan the scene with the goggles to detect an object was due to the relatively small area of the field of optogenetic activation.

The three visual tests were performed in an indoor laboratory (Streetlab, http://www.streetlab-vision.com/?lang=en) according to a preestablished protocol. We also examined whether the patient could recognize patterns during locomotion outside on the street. In the stimulated monocular condition but not in the natural binocular condition, the patient spontaneously reported identifying crosswalks and he could count the number of white stripes. Subsequently, the patient testified to a major improvement in daily visual activities, such as detecting a plate, mug or phone, finding a piece of furniture in a room or detecting a door in a corridor but only when using the goggles. Thus, treatment by the combination of an optogenetic vector with light-stimulating goggles led to a level of visual recovery in this patient that was likely to be of meaningful benefit in daily life.

Interestingly, while performing the psychophysical tests under stimulated monocular condition, the patient reported 'vertical vibrations' when perceiving an object (Supplementary Video 1). The patient did not report vibrations when wearing the light-stimulating goggles before the injection, suggesting that optogenetic activation was responsible for this phenomenon. We hypothesize that the vibrations are caused by the use of an event-based camera that provides localized light pulses at each pixel where the camera detects changes in contrast. When the camera scans through an object, synchronized light pulses are sent to the eye, which may be perceived by the individual as 'vibrations'. We have no explanation yet why the patient reported the vibrations as 'vertical'.

The red fluorescent protein tdTomato encoded by the injected vector could, in theory, be visualized by a scanning laser ophthalmoscope. However, visualization of red fluorescent probes using scanning laser ophthalmoscopy is not yet approved for clinical use. Should this be approved in the future, direct visualization of the cells expressing the fusion protein ChrimsonR- tdTomato could prove particularly useful to monitor vector transfection and to individually tailor the size and location of the light beam projected by the device.

Taken together, the psychophysical and neurophysiological evidence presented in this article suggest that the optogenetic stimulation of human retinal ganglion cells by a light-projection system linked to a camera is a promising way to partially restore vision in blind patients affected with advanced RP.

\section{Online content}

Any methods, additional references, Nature Research reporting summaries, source data, extended data, supplementary information, acknowledgements, peer review information; details of author contributions and competing interests; and statements of data and code availability are available at https://doi.org/10.1038/ s41591-021-01351-4.

Received: 23 November 2020; Accepted: 13 April 2021; Published online: 24 May 2021

\section{References}

1. Kajiwara, K., Berson, E. L. \& Dryja, T. P. Digenic retinitis pigmentosa due to mutations at the unlinked peripherin/RDS and ROM1 loci. Science 264, 1604-1608 (1994).

2. Russell, S. et al. Efficacy and safety of voretigene neparvovec (AAV2-hRPE65v2) in patients with RPE65-mediated inherited retinal dystrophy: a randomised, controlled, open-label, phase 3 trial. Lancet 390, 849-860 (2017).

3. $\mathrm{Bi}, \mathrm{A}$. et al. Ectopic expression of a microbial-type rhodopsin restores visual responses in mice with photoreceptor degeneration. Neuron 50, 23-33 (2006).

4. Busskamp, V. et al. Genetic reactivation of cone photoreceptors restores visual responses in retinitis pigmentosa. Science 329, 413-417 (2010).

5. Lagali, P. S. et al. Light-activated channels targeted to ON bipolar cells restore visual function in retinal degeneration. Nat. Neurosci. 11 667-675 (2008)

6. Roska, B. \& Sahel, J.-A. Restoring vision. Nature 557, 359-367 (2018).

7. Sahel, J.-A., Bennett, J. \& Roska, B. Depicting brighter possibilities for treating blindness. Sci. Transl. Med. 11, eaax2324 (2019).

8. Sahel, J.-A. \& Roska, B. Gene therapy for blindness. Annu. Rev. Neurosci. 36, 467-488 (2013)

9. Scholl, H. P. N. et al. Emerging therapies for inherited retinal degeneration. Sci. Transl. Med. 8, 368rv6 (2016).

10. Gauvain, G. et al. Optogenetic therapy: high spatiotemporal resolution and pattern discrimination compatible with vision restoration in non-human primates. Commun. Biol. 4, 125 (2021) 
11. Picaud, S. et al. The primate model for understanding and restoring vision. Proc. Natl Acad. Sci. USA 116, 26280-26287 (2019).

12. Dalkara, D. et al. In vivo-directed evolution of a new adeno-associated virus for therapeutic outer retinal gene delivery from the vitreous. Sci. Transl. Med. 5, 189 ra76 (2013).

13. Klapoetke, N. C. et al. Independent optical excitation of distinct neural populations. Nat. Methods 11, 338-346 (2014).

14. Posch, C., Matolin, D. \& Wohlgenannt, R. A QVGA 143 dB dynamic range frame-free PWM image sensor with lossless pixel-level video compression and time-domain CDS. IEEE J. Solid-State Circuits 46, 259-275 (2011).

15. Jabs, D. A., Nussenblatt, R. B. \& Rosenbaum, J. T., Standardization of Uveitis Nomenclature (SUN) Working Group. Standardization of uveitis nomenclature for reporting clinical data. Results of the First International Workshop. Am. J. Ophthalmol. 140, 509-516 (2005).

16. Trusko, B. et al. The Standardization of Uveitis Nomenclature (SUN) Project. Development of a clinical evidence base utilizing informatics tools and techniques. Methods Inf. Med. 52, 259-265 (2013).

17. Francois, J., Stefens, R. \& Derouck, A. Electro-retino-encephalography in pigmented retinopathy. Ann. Ocul. (Paris) 187, 908-937 (1954).

18. Streifler, M. \& Landau, J. Electrical brain potentials in retinitis pigmentosa and familial hemeralopia. Ophthalmologica 130, 116-127 (1955).

19. Krill, A. E. \& Stamps, F. W. The electroencephalogram in retinitis pigmentosa. Am. J. Ophthalmol. 49, 762-773 (1960).

20. Ebe, M., Mikami, T. \& Ito, H. Clinical evaluation of electrical responses of retina and visual cortex to photic stimulation in ophthalmic diseases. Tohoku J. Exp. Med. 84, 92-103 (1964).

21. Gillespie, F. D. \& Dohogne, V. Z. Electro-encephalograms in retinitis pigmentosa. Am. J. Ophthalmol. 57, 1045-1050 (1964).

22. Parisi, V. et al. Impact of regional retinal responses on cortical visually evoked responses: multifocal ERGs and VEPs in the retinitis pigmentosa model. Clin. Neurophysiol. 121, 380-385 (2010).

23. Hamilton, R. et al. VEP estimation of visual acuity: a systematic review. Doc. Ophthalmol. 142, 25-74 (2021).

24. Alexander, K. R., Rajagopalan, A. S., Seiple, W., Zemon, V. M. \& Fishman, G. A. Contrast response properties of magnocellular and parvocellular pathways in retinitis pigmentosa assessed by the visual evoked potential. Invest. Ophthalmol. Vis. Sci. 46, 2967-2973 (2005).

25. Paranhos, F. R., Katsum, O., Arai, M., Nehemy, M. B. \& Hirose, T. Pattern reversal visual evoked response in retinitis pigmentosa. Doc. Ophthalmol. 96, 321-331 (1998).
26. Myers, M. H., Iannaccone, A. \& Bidelman, G. M. A pilot investigation of audiovisual processing and multisensory integration in patients with inherited retinal dystrophies. BMC Ophthalmol. 17, 240 (2017).

27. Bottari, D. et al. Sight restoration after congenital blindness does not reinstate alpha oscillatory activity in humans. Sci. Rep. 6, 24683 (2016).

28. Feng, W., Störmer, V. S., Martinez, A., McDonald, J. J. \& Hillyard, S. A. Involuntary orienting of attention to a sound desynchronizes the occipital alpha rhythm and improves visual perception. Neuroimage 150, 318-328 (2017).

29. Vanni, S., Revonsuo, A. \& Hari, R. Modulation of the parieto-occipital alpha rhythm during object detection. J. Neurosci. 17, 7141-7147 (1997).

30. Van Dijk, H., Schoffelen, J.-M., Oostenveld, R. \& Jensen, O. Prestimulus oscillatory activity in the alpha band predicts visual discrimination ability. $J$. Neurosci. 28, 1816-1823 (2008).

31. Romei, V., Gross, J. \& Thut, G. On the role of prestimulus alpha rhythms over occipito-parietal areas in visual input regulation: correlation or causation? $\mathrm{J}$. Neurosci. 30, 8692-8697 (2010).

32. Wyart, V. \& Tallon-Baudry, C. Neural dissociation between visual awareness and spatial attention. J. Neurosci. 28, 2667-2679 (2008).

33. Thut, G., Nietzel, A., Brandt, S. A. \& Pascual-Leone, A. $\alpha$-Band electroencephalographic activity over occipital cortex indexes visuospatial attention bias and predicts visual target detection. J. Neurosci. 26 9494-9502 (2006).

34. Freunberger, R., Klimesch, W., Griesmayr, B., Sauseng, P. \& Gruber, W. Alpha phase coupling reflects object recognition. Neuroimage 42, 928-935 (2008).

35. Romei, V. et al. Spontaneous fluctuations in posterior $\alpha$-band EEG activity reflect variability in excitability of human visual areas. Cereb. Cortex $\mathbf{1 8}$ 2010-2018 (2008).

36. Vandewalle, G. et al. Light modulates oscillatory alpha activity in the occipital cortex of totally visually blind individuals with intact non-image-forming photoreception. Sci. Rep. 8, 16968 (2018).

37. Ergenoglu, T. et al. Alpha rhythm of the EEG modulates visual detection performance in humans. Brain Res. Cogn. Brain Res. 20, 376-383 (2004)

38. Zumer, J. M., Scheeringa, R., Schoffelen, J.-M., Norris, D. G. \& Jensen, O. Occipital alpha activity during stimulus processing gates the information flow to object-selective cortex. PLoS Biol. 12, e1001965 (2014).

Publisher's note Springer Nature remains neutral with regard to jurisdictional claims in published maps and institutional affiliations.

(C) The Author(s), under exclusive licence to Springer Nature America, Inc. 2021 


\section{Methods}

Study design. PIONEER is a multicenter, phase $1 / 2$ a, open-label, nonrandomized, dose-escalation study to evaluate the safety and tolerability of an adeno-associated viral vector, GS030-DP, administered via a single intravitreal injection to the worse-seeing eye of patients with nonsyndromic RP, and ensuing light stimulation via light-stimulating goggles (GS030-MD). The secondary objective of the study is to evaluate visual and visuomotor function with and without the light-stimulating goggles. The clinical trial protocol, including patient selection criteria, is provided in the Supplementary Text. Before initiation, the PIONEER study protocol was approved by the Agence Nationale de Sécurité du Médicament et des Produits de Santé, the US Food and Drug Administration, the Medicines and Healthcare products Regulatory Agency and the following national/local ethics committees and institutional review board: Comité de Protection des Personnes Île-de-France III; North East-York Research Ethics Committee; and Human Research Protection Office at the University of Pittsburgh. Study participants provided written informed consent before enrollment, according to CARE guidelines and in compliance with the Declaration of Helsinki 2013. The individual participating in the experiments described in this report was a 58-year-old man. The study design includes three dose-escalation cohorts $\left(5.0 \times 10^{10}, 1.5 \times 10^{11}\right.$ and $5.0 \times 10^{11}$ viral genomes per eye) of three participants each and an extension cohort treated at the highest tolerated dose. After each cohort is completed, a data safety monitoring board reviews safety data and recommends escalation to the next dose. As of the end of 2020 , seven patients had received a single intravitreal injection of GS030-DP in their worse-seeing eye: three patients in each of the first two cohorts and one patient in the third cohort. Because of COVID-19, only one patient from the first cohort, the patient described in this article, could perform sustained $(n=15)$ postinjection training sessions. So far, the pandemic has prevented any functional assessment of the combined therapy in the other treated patients. Genotypic studies were performed in all patients of the study. The reported patient has two pathogenic alleles on the USH2A gene (NM_206933.2) that were identified by targeted next-generation sequencing ${ }^{39}$ and segregation analysis using samples from his parents. The first pathogenic allele is on exon 13 c.2299del p.(Glu767Serfs $\left.{ }^{\star} 21\right)^{40}$ and was inherited from his father. The second pathogenic allele is a complex allele with exon 22 c. 4714 C > T p.Leu1572Phe ${ }^{41}$ and exon 50 c.9882 C>G p.Cys3294Trp ${ }^{42}$, which was inherited from his mother.

Assessment of intraocular inflammation. We used standardized assessment of intraocular inflammation according to the international guidelines of the Standardization of Uveitis Nomenclature Working Group, such as the assessment of the anatomic location, severity and clinical evolution (activity) of anterior and intermediate uveitis ${ }^{15,16,43}$ (Supplementary Text)

GS030-DP. GS030-DP consists of a serotype $2.7 \mathrm{~m} 8$ (ref. ${ }^{12}$ ) adeno-associated viral vector expressing the fusion protein ChrimsonR-tdTomato ${ }^{13}$ under the control of the CAG promoter (AAV2.7m8-CAG-ChrimsonR-tdTomato). A human growth hormone 1 polyadenylation signal sequence was inserted in the $3^{\prime}$ end of the construct. Similar to other adeno-associated viruses, GS030-DP is replication-defective and contains single-stranded DNA. Capsid $2.7 \mathrm{~m} 8$ was created by inserting a 10-mer peptide in the capsid of AAV2 (ref. ${ }^{12}$ ).

AAV2.7m8-CAG-ChrimsonR-tdTomato was suspended in phosphate buffer including $0.001 \%$ Pluronic F-68. The GS030-DP suspension was dispensed into individual vials and stored at $\leq-70^{\circ} \mathrm{C}$ until use. An aliquot of $100 \mu$ of GS030-DP $\left(5.0 \times 10^{10}\right.$ vector genomes) was administered via intravitreal injection once into the worse-seeing eye.

GS030-MD. The external medical device GS030-MD consists of two hardware units connected by a high-speed link. The first unit (head unit) is a pair of goggles hosting a camera and a light-projection system. The goggles are connected to the second unit (processing unit) that runs the software (Extended Data Fig. 1).

Each pixel of the camera ${ }^{14}$ continuously records the light intensity in a local region of the natural scene, with a dynamic range of $120 \mathrm{~dB}$. If the change in light intensity crosses a threshold, the camera signals an event, which is represented by the coordinates of the pixel at which the change was recorded. The events are asynchronous in time and 33,346 camera pixels are used for event detection. The camera pixels are then mapped to the pixels of the light-projection system, which projects the image onto a circular retinal area of $10^{\circ}$ of visual angle. A light pulse at a given projected image pixel, which corresponds to an event, has an onset precision of $0.694 \mathrm{~ms}(1,440 \mathrm{~Hz})$ and is $16.6-\mathrm{ms}$ long $(60 \mathrm{~Hz})$. The image is binary, that is, each individual pixel is either ON or OFF. A stimulus pixel is ON in a given image if the corresponding camera pixel signaled an event; otherwise it is OFF. The light-projection system uses a light-emitting diode light source (595-nm peak wavelength, 15-nm half width, tailored to activate ChrimsonR close to the peak of its action spectrum) that is projected onto an array of individually switchable micromirrors (digital micromirror device) mounted on the goggles. A given micromirror of the array can be either in the ON or OFF position, leading to light reflectance or no light reflectance. This results in a binary pixelated image stream that is projected onto the retina. Note that the stimulation at different contrasts is not the same since the probability of an event being generated by a pixel in the camera varies with the contrast of the stimulus ${ }^{44}$. The light-stimulating goggles output a maximum radiative flux of $1.3 \mathrm{~mW}$, which corresponds to a retinal irradiance of $13 \mathrm{~mW} \mathrm{~cm}^{-2}\left(4 \times 10^{16}\right.$ photons $\left.\mathrm{cm}^{-2} \mathrm{~s}^{-1}\right)$ for a pupil size of $6 \mathrm{~mm}$. This is 51 times below thermal safety thresholds set by ophthalmological standards ${ }^{45}$. Note that the pupil of the patient was not dilated when wearing the goggles and the assumed 6-mm pupil size corresponding to a dilated pupil was only used to calculate an upper limit for retinal irradiance. The light-stimulating goggles can create a retinal irradiance ranging from a minimum of $4 \times 10^{14}$ photons $\mathrm{cm}^{-2} \mathrm{~s}^{-1}$ to $4 \times 10^{16}$ photons $\mathrm{cm}^{-2} \mathrm{~s}^{-1}$. This range was chosen because preclinical studies in nonhuman primates indicated that ChrimsonR-expressing foveal retinal ganglion cells were activated starting at $10^{15}$ photons $\mathrm{cm}^{-2} \mathrm{~s}^{-1}$ (ref. ${ }^{10}$ ) The maximal corneal irradiance of the light-stimulating goggles is $4.7 \mathrm{~mW} \mathrm{~cm}^{-2}$, which is 850 times below the thresholds set by ophthalmological standards for the anterior segment and 127 times below the thresholds set by the American National Standard for Safe Use of Lasers Z136.1 standard ${ }^{46}$ for pigmented iris illumination. When using 595-nm light, the photochemical damage threshold of the retina ${ }^{47}$, expressed in retinal irradiance $\left(E^{\max }\right)$ at $6-\mathrm{mm}$ pupil size and at $t \geq 10,000 \mathrm{~s}$, is $880 \mathrm{~mW} \mathrm{~cm}^{-2}$. This value is 67 times higher than the maximal retinal irradiance of $13 \mathrm{~mW} \mathrm{~cm}^{-2}$ produced by the light-stimulating goggles at the same pupil size. As a precautionary measure and with approval from regulatory agencies, the use of the device was limited to $4 \mathrm{~h}$ per day in the context of this clinical trial. The light-stimulating goggles were classified by the IEC 62471 standard ${ }^{48}$ in the risk group 'exempt', indicating that they do not constitute a photobiological hazard. The maximum irradiance provided by the device was well tolerated by the patient. The patient tested the goggles three times before the injection was administered and did not report any photophobia.

Visual training. Training of participants was performed at Streetlab, a center of naturalistic vision rehabilitation platforms dedicated to the evaluation of vision and training in low-vision patients. The first step of visual training was to teach the patient, who had lost vision two years before enrollment in the clinical trial and had divergent strabismus in his treated eye, to become aware of the direction of his gaze and to control his eye movements to be able to look straight into the light beam projected by the goggles. With a visual acuity limited to light perception, the patient was able to learn how to align his gaze with the light beam of the goggles even before any improvement in vision. Visuomotor exercises were initially performed without the goggles, then with the goggles in simple exercises and eventually in daily life (Extended Data Fig. 7): (1) oculomotor exercises without goggles. Each visit started with ocular relaxation exercises, which were followed by fixation exercises, ocular pursuit exercises and eye-hand coordination exercises; (2) oculomotor exercises with goggles (simple exercises). Training with the goggles was initiated four and a half months after the injection and included four types of exercises: (a) camera-target alignment exercises. The objective was to make the patient aware of the position of the camera on the goggles; (b) eye-beamtarget alignment exercises. The objective was to train the patient to look with his treated eye straight into the light beam projected by the goggles once a target was aligned with the camera; (c) scanning exercises. The objective was to learn head-scanning and eye-movement strategies during target search with the camera of the goggles. Using fine head movements, the patient had to localize a contrasting target, follow its contours and identify its shape. With the same scanning technique, the patient also had to locate multiple targets one after the other; (d) eye-hand coordination exercises. The objective was to learn how to associate the visual perception of a target with its physical location. The patient first had to visually locate a target and then touch it. In addition, he had to follow the edge of a target with the camera, identify its shape and eventually touch the target; (3) Oculomotor exercises with goggles (daily life exercises). Daily life exercises were conducted at Streetlab starting seven months after the injection. Ten months after the injection, the patient agreed to use the goggles during locomotion outside.

Visual tests. The first two functional vision tests described in the main text each lasted for $30 \mathrm{~min}$ and the trials were randomized according to object type, contrast and location. Before each of the two tests, the objects and their possible locations were verbally described to the patient once. Before each trial, the patient sitting at the table had to close his eyes when the experimenter prepared the test to prevent the patient from localizing the object(s) in advance. In addition, the experimenter was careful not to make any noise when placing the object(s) on the table. Foam was placed under each object to avoid any sound during placement. The experimenter always stood on the same side of the patient when placing objects on the table. After placing the object, the experimenter moved back to their original position about $1 \mathrm{~m}$ away from the patient. For trials without objects, the experimenter pretended to put something on the table. The objects and settings specific to the two psychophysical tests were not used during the visual training sessions. However, the patient was trained to implement strategies to perceive, locate and touch objects of different sizes, shapes and contrasts.

Image contrast. Image contrast was assessed using two measures. Michelson contrast was measured as $\left(I_{\max }-I_{\min }\right) /\left(I_{\max }+I_{\min }\right)$, where $I_{\max }$ is the intensity of the table and $I_{\min }$ that of the object. RMS contrast was measured as the s.d. of the image pixel intensities in a circle $21 \mathrm{~cm}$ in diameter, which included the object in the middle. The local illumination associated with the different RMS values was as 
follows. First test (notebook): RMS contrast $=0.80$, local luminance $51.90 \mathrm{Cd} \mathrm{m}^{-2}$. RMS contrast $=0.53$, local luminance $59.23 \mathrm{Cd} \mathrm{m}^{-2}$; RMS contrast $=0.41$, local luminance $63.94 \mathrm{Cd} \mathrm{m}^{-2}$. First test (staple box): RMS contrast $=0.21$, local luminance $94.92 \mathrm{Cd} \mathrm{m}^{-2}$; RMS contrast $=0.16$, local luminance $93.83 \mathrm{Cd} \mathrm{m}^{-2}$; RMS contrast $=0.13$, local luminance $94.14 \mathrm{Cd} \mathrm{m}^{-2}$. Second test (tumbler): RMS contrast $=0.41$, local luminance $82.91 \mathrm{Cd} \mathrm{m}^{-2} ;$ RMS contrast $=0.33$, local luminance $85.11 \mathrm{Cd} \mathrm{m}^{-2}$; RMS contrast $=0.29$, local luminance $83.28 \mathrm{Cd} \mathrm{m}^{-2}$. Third test (tumbler): RMS contrast $=0.33$, local luminance $85.11 \mathrm{Cd} \mathrm{m}^{-2}$.

Extracranial EEG recordings. Visual detection task. The third visual test was adapted to simultaneous behavioral and EEG recordings (Fig. 1). The task was to detect the presence of an object (tumbler) placed $80 \mathrm{~cm}$ in front of the patient (Fig. 1a,c; Michelson contrast $=55 \%$ ). Unlike the other two visual tasks, object location was fixed and the patient was informed before the beginning of the experiment that the object position would not change throughout the experiment. The patient acknowledged the presence or absence of the object by means of a two-key response pad but he was not forced to answer. The protocol included a total of 183 randomized object/no-object trials distributed across three conditions: natural binocular; natural monocular; and stimulated monocular (60, 60 and 63 trials, respectively). The experiment was split into two sessions of approximately 70 min each (see Fig. $1 \mathrm{~b}$ for the detailed protocol of each session). Each trial lasted $20 \mathrm{~s}$. During the first $5 \mathrm{~s}$, the patient was instructed to close his eyes while the experimenter silently placed or did not place an object on the table (thus avoiding visual and auditory cues). The patient was then instructed to open his eyes, search for the object and provide an answer within $15 \mathrm{~s}$. The between-trial time interval was set according to the participant's pace.

EEG data acquisition and preprocessing. A 64-channel EEG cap with passive electrodes (Waveguard original, standard 10/10 system; ANT Neuro) connected to an eego mylab amplifier (ANT Neuro) was used to record time-dependent cortical activity (Fig. 1c). EEG signals were recorded from 48 electrodes (out of 64) distributed at a higher density in the occipital area. Data were acquired with the eego software v1.9.1 (ANT Neuro) and preprocessed with the EEGLAB toolbox of MATLAB (R2019a-20a) via the following pipeline. Raw recordings were first highand low-pass-filtered ( 1 and $40 \mathrm{~Hz}$, respectively). Artifactual channels were then removed and interpolated. Independent component analysis was performed to decompose the EEG signals into statistically independent components, which were then labeled using the ICLabel algorithm ${ }^{49}$ to probabilistically assign the origin of their signals to brain, muscles, heart, eyes or artifactual sources. Finally, the most likely brain-related independent components were selected and used to reconstruct data into the electrode space before the analyses.

Spectral analysis of EEG data and decoding. Spectral EEG analyses were conducted to identify power spectrum signatures in the alpha frequency range $(8-14 \mathrm{~Hz})$, which is associated with the intensity of visual processing in the occipital region ${ }^{28,36}$. A binary decoder was trained to discriminate object versus no-object trials based on the alpha-band power amplitudes over the occipital channels $\left(\mathrm{O}_{z}, \mathrm{O}_{1}\right.$, and $\mathrm{O}_{2}$; Fig. 1c). EEG data recorded during the eyes-open periods of the stimulated condition were used for training, after signal power amplitude normalization across trials (frequency $\times$ channel pairwise, independently) for feature comparability. The Fisher score was used to quantify the discriminant power of each spectral feature:

$$
\text { Fisher score }=\frac{\sum_{c}(\mu(c)-\mu)^{2}}{\sum_{c} \sigma(c)}
$$

where $\mu(c)$ and $\mu$ indicate the means of the observations within the class $c$ and over all classes, respectively and $\sigma(c)$ is the variance of the observations within the class $c$. By computing the ratio between the separability of means across all classes and the overall spread around their means, the Fisher score provided a discriminability index between classes (two in the case of the binary decoder used in this study).

The six most informative features (that is, the features with the highest Fisher scores) were fed into the decoder in increasing number, leading to multiple instances of the decoder. For each instance, $k$-fold cross-validation (20-fold) and leave-one-out cross-validation procedures were then used to assess the decoding accuracy on testing samples (that is, data unseen by the decoder during training). Finally, the optimal number of features was determined based on the instance with the best decoding accuracy. As a control, the decoder was also trained and tested on data extracted from the eyes-closed periods of the stimulated condition and from the eyes-open and eyes-closed periods of the natural conditions for classification comparison purposes.

Statistical analyses. Statistical analyses of behavioral data were conducted in R v.4.0.3. Likelihood-ratio tests for the effects of explanatory variables in multivariable logistic regressions were used to compare the data in Tables 1-3. A likelihood-ratio test was used to check whether the patient's counting performance was above chance level in Table 2. Statistical analyses of EEG data were conducted in JASP v.0.11.1. All tests on EEG data assumed independence between the samples of each distribution and normality was assessed using the Shapiro-Wilk test to apply either parametric or nonparametric tests. A one-sided, nonparametric test (one-sample Wilcoxon signed-rank test) was employed for each decoder separately to determine whether classification accuracy was above chance level (50\%). A two-sided nonparametric test (Mann-Whitney $U$-test) was used to investigate the spectral power modulation in the object and no-object trials.

Reporting Summary. Further information on research design is available in the Nature Research Reporting Summary linked to this article.

\section{Data availability}

All requests for the raw and analyzed data are promptly reviewed by GenSight Biologics to verify if they are subject to any intellectual property or confidentiality obligations. Patient-related data not included in the paper were generated as part of clinical trials and may be subject to patient confidentiality. Any data that can be shared will be released via a material transfer agreement. All raw and analyzed image data can be found at https://passageinnovation-my. sharepoint.com/:f:/g/personal/mtaiel_gensight-biologics_com/ EkUITiEa4AxNs_YryLq7fT8BGpdYkXZMWWtDK6Wg-fcQfA.

\section{Code availability}

The code for the EEG data processing and spectral analysis is available at https://github.com/JBDSA/OptoRehabEEG.

\section{References}

39. Audo, I. et al. Development and application of a next-generation-sequencing (NGS) approach to detect known and novel gene defects underlying retinal diseases. Orphanet J. Rare Dis. 7, 8 (2012).

40. Eudy, J. D. et al. Mutation of a gene encoding a protein with extracellular matrix motifs in Usher syndrome type IIa. Science 280, 1753-1757 (1998).

41. Song, J. et al. High-throughput retina-array for screening 93 genes involved in inherited retinal dystrophy. Invest. Ophthalmol. Vis. Sci. 52, 9053-9060 (2011).

42. Nishiguchi, K. M. et al. Whole genome sequencing in patients with retinitis pigmentosa reveals pathogenic DNA structural changes and NEK2 as a new disease gene. Proc. Natl Acad. Sci. USA 110, 16139-16144 (2013).

43. Nussenblatt, R. B., Palestine, A. G., Chan, C. C. \& Roberge, F. Standardization of vitreal inflammatory activity in intermediate and posterior uveitis. Ophthalmology 92, 467-471 (1985).

44. Posch, C. \& Matolin, D. Sensitivity and uniformity of a $0.18 \mu \mathrm{m}$ CMOS temporal contrast pixel array. in Proc. 2011 IEEE International Symposium of Circuits and Systems (ISCAS) 1572-1575 (IEEE, 2011).

45. International Standards Organization. ISO 15004-2:2007: ophthalmic instruments-Fundamental requirements and test methods-Part 2: light hazard protection. https://www.iso.org/standard/38952.html (2007).

46. American National Standards Institute. ANSI Z136.1-2014: American National Standard for Safe Use of Lasers (Laser Institute of America, 2014); https://webstore.ansi.org/Standards/LIA/ANSIZ1362014

47. International Commission on Non-Ionizing Protection. Guidelines on limits of exposure to broad-band incoherent optical radiation ( 0.38 to $3 \mu \mathrm{M})$. Health Phys. https://www.icnirp.org/cms/upload/publications/ICNIRPbroadband.pdf (1997).

48. International Electrotechnical Commission. Safety of Laser Products-Part 1: Equipment Classification and Requirements 3rd edn (ISO, 2014).

49. Pion-Tonachini, L., Kreutz-Delgado, K. \& Makeig, S. ICLabel: an automated electroencephalographic independent component classifier, dataset, and website. Neuroimage 198, 181-197 (2019).

\section{Acknowledgements}

The PIONEER study group members were H. Akolkar, F. Arcizet, E. Bamberg, S. Bentobji, S. Bertin, E. Bochin, E. Boyden, A. Chaffiol, G. Chenegros, C. Ciavrs-Roux, C. Coen, D. Dagostinoz, M.-C. Despiau, M. Desrosiers, C. Devisme, G. Gauvain, J.-F. Girmens, A. Grimaud, R. Hosseini, C. Jaillard, L. Karbunarevic, J. Labbe, M. Laurent, J. P. Marques, O. Marre, G. Martin, R. Mecheri, N. Messeca, M. Michaelides, S. Mohand-Said, P. Pouget, P. Queromes, M. Roux, S. Sancho, I. Scarabin, P. Shabestary, W. Smith, A. Tufail, A. Webster. We thank M. Cattaneo for help with statistical analysis. We thank R. Benosman and his lab for their contribution to the development of the stimulation goggles and to the preclinical development. The study was supported by the following sources of funding: GenSight Biologics; French Programme Investissements d'Avenir IHU FOReSIGHT, no. ANR-18-IAHU-01 (J.-A.S., A.A. and I.A.) and RHU LIGHT4DEAF no. ANR-15-RHU-0001 (J.-A.S. and I.A.), BPI France (grant no. 2014-PRSP-15 to J.-A.S.), Foundation Fighting Blindness (J.-A.S. and I.A.) and Fédération des Aveugles de France and French National Research Agency (no. ANR-18-CHIN-0002 to A.A.).

\section{Author contributions}

J.-A.S. designed the study and wrote the paper. E.B.-S. collected the data. C.P. designed the functional vision tests, collected the data, drew Extended Data Figs. 7-9, recorded 
and edited the video and wrote the paper. A.A. designed the EEG experiment, collected and analyzed the data, drew Figs. 1 and 2 and wrote the paper. F.G. designed the software for the medical device and wrote the paper. J.N.M. and S.D.E. collected the data. A.D. designed the EEG experiment, collected and analyzed the data, drew Figs. 1 and 2 and wrote the paper. J-B.d.S.A. designed the EEG experiment, collected and analyzed the data, drew Figs. 1 and 2 and wrote the paper. C.d.M. designed and collected the data for the vision tests. E.G. designed the functional vision tests. I.A. collected and analyzed the data. J.D. contributed to the preclinical development. S.P. contributed to the preclinical development. D.D. contributed to the preclinical development and wrote the paper. L.B. analyzed the data, drew the figures and wrote the paper. M.T. supervised the study, monitored the safety data and wrote the paper. B.R. analyzed the data and wrote the paper.

\section{Competing interests}

F.G., L.B. and M.T. are employees of GenSight Biologics. B.R. is the Chair of the Scientific Advisory Board of GenSight Biologics and is a board member of and has financial interest in Arctos Medical AG. J.-A.S. has personal financial interests in GenSight Biologics, Pixium Vision, SparingVision, Prophesee, Tilak, VegaVect, NewSight and Chronolife. E.B.-S. is a consultant for GenSight Biologics. D.D. was a consultant for GenSight Biologics, is a cofounder and acting Chief Strategy Officer of Gamut Tx.
D.D. is an inventor on a patent of adeno-associated virus virions with variant capsid and methods of use thereof with royalties paid to Adverum (WO2012145601 A2). S.P. owns shares in, received consultant fees from and has filed a patent (WO 2017/187272) licensed to GenSight Biologics. S.P. has financial interests in Pixium Vision, Gamut, Iconeus, Chronolife, Neurallys and Prophesee. I.A. is a consultant for Novartis, Biogen and SparingVision and a cofounder of Gamut Tx. The other authors declare no competing interests.

\section{Additional information}

Extended data is available for this paper at https://doi.org/10.1038/s41591-021-01351-4.

Supplementary information The online version contains supplementary material available at https://doi.org/10.1038/s41591-021-01351-4.

Correspondence and requests for materials should be addressed to J.-A.S. or B.R.

Peer review information Nature Medicine thanks Bart Leroy, Ione Fine and the other, anonymous, reviewer(s) for their contribution to the peer review of this work. Jerome Staal was the primary editor on this article and managed its editorial process and peer review in collaboration with the rest of the editorial team.

Reprints and permissions information is available at www.nature.com/reprints. 
a

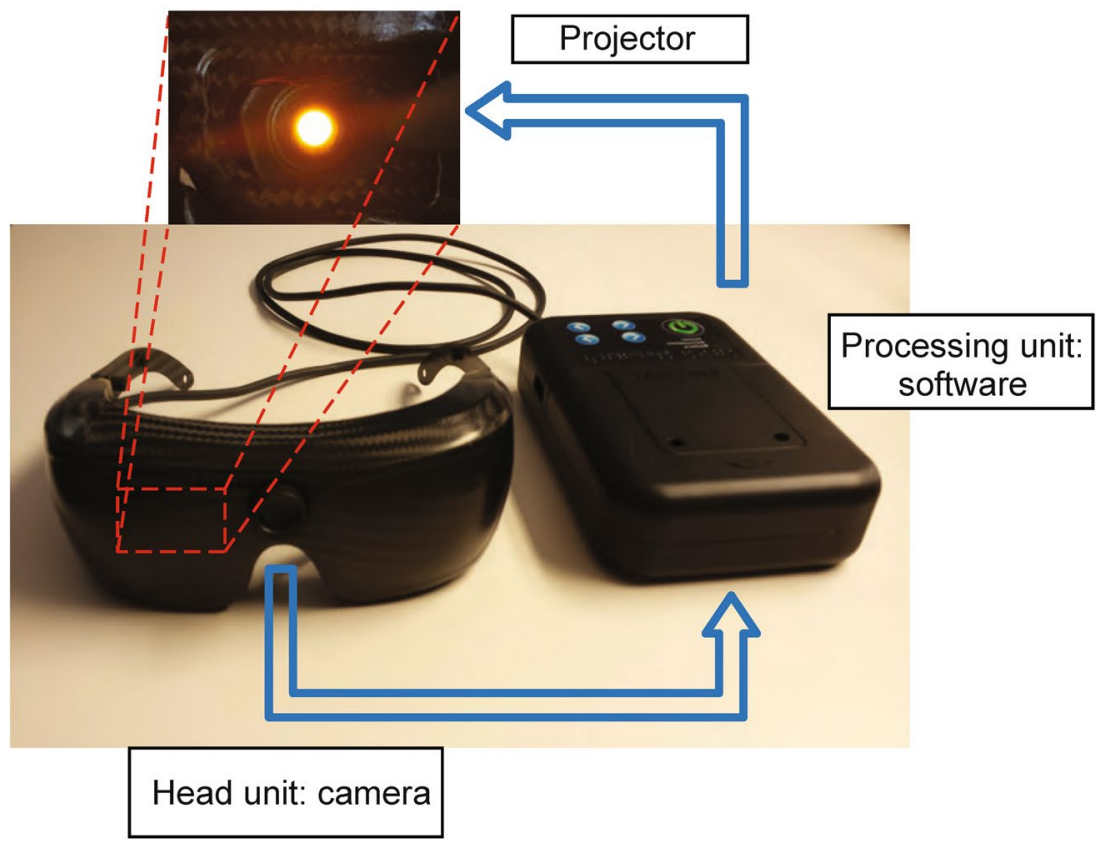

b

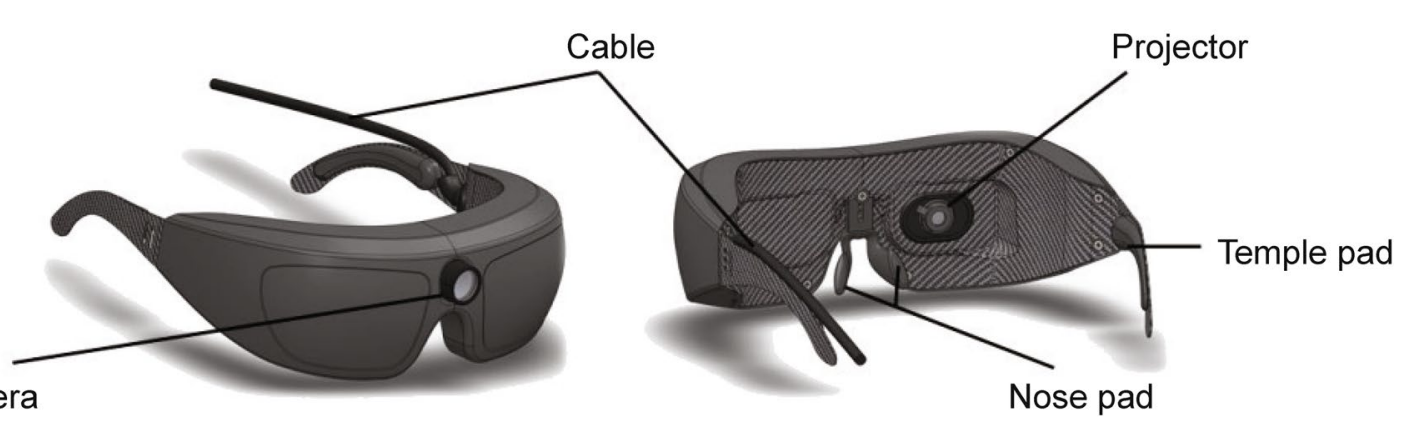

Extended Data Fig. 1 | Light-stimulating goggles. a. GSO3O-MD consists of two units connected by a high-speed link. The head unit (left) hosts the camera which acquires the natural scene in a stream of asynchronous address-events representing pixel coordinates of local relative light intensity changes. The processing unit (right) encodes the visual stream in real time and creates binary images that are sent to the projector in the head unit. The projector, which is also mounted on the head unit (top), is placed in front of the eye. $\mathbf{b}$. Front and rear view of the head unit. The camera is placed at the center facing the outside world. The projector is placed in front of the treated eye, facing the eye, and can be moved horizontally so as to be placed precisely in front of the treated eye's pupil, since inter-pupillary distance varies between patients. 


\section{a}

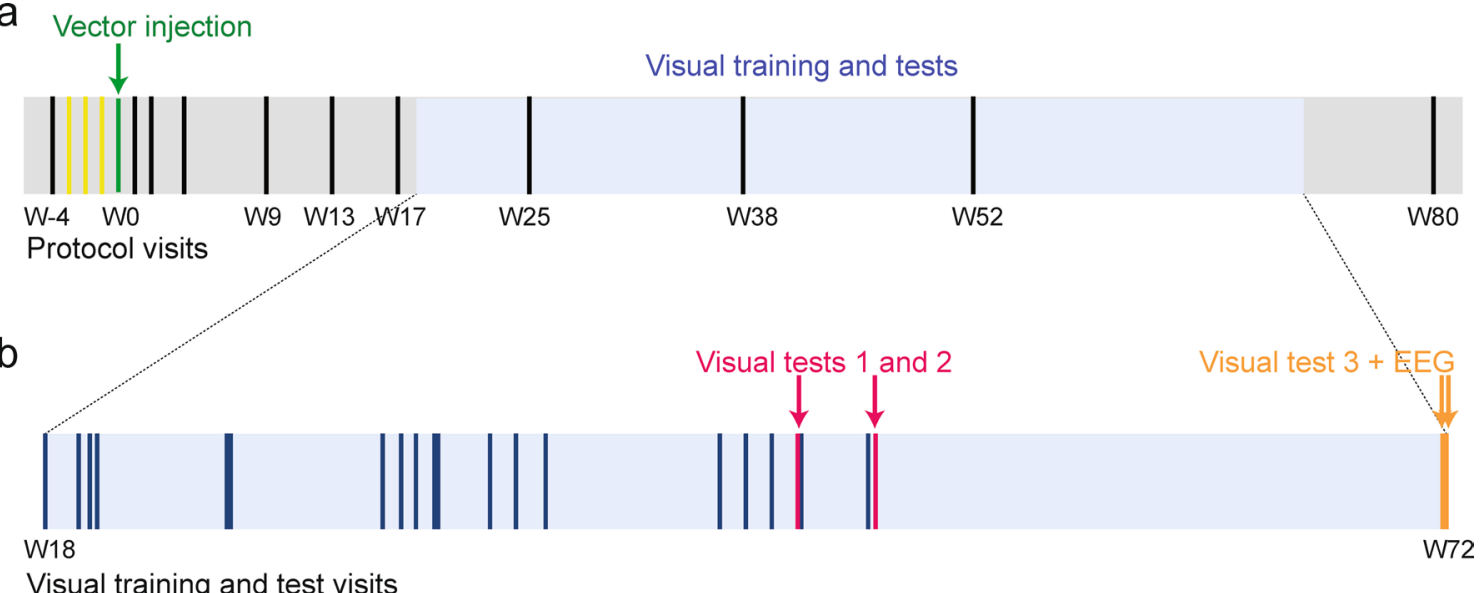

Extended Data Fig. 2 | Timeline of protocol visits, visual training visits, and test visits. a. Timeline of protocol visits. Each vertical line corresponds to a visit described in the clinical trial protocol. The visit (week $(W) 0$ ) at which the subject was injected is shown in green. The light-stimulating goggles were tested before injection on $\mathrm{W}-3, \mathrm{~W}-2$, and $\mathrm{W}-1$, shown in yellow. Protocol visits included ocular and general examinations. The time period at which visual training and the three visual tests occurred is shown in light blue. $\mathbf{b}$. Timeline of visual training and visual test visits, which corresponds to the light blue region on a. Each dark blue line corresponds to a visual training visit (19 visits, thick lines indicate two closely spaced visits). Visual tests 1 and 2 were both run on the two visits shown in red, visual test 3 and EEG (electroencephalography) were run on the two visits shown in orange. 


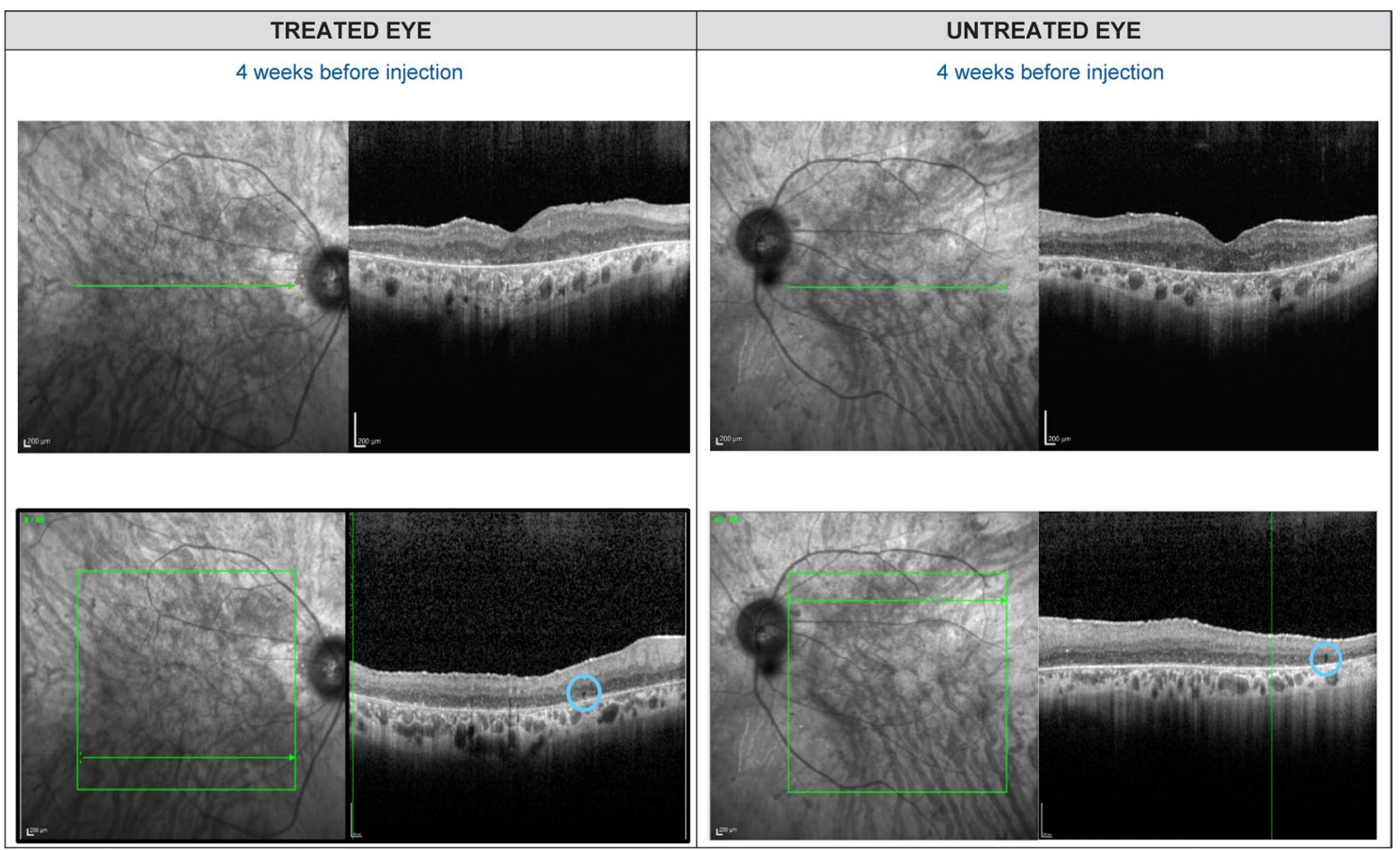

Extended Data Fig. 3 | OCT imaging of the retina 4 weeks before injection. OCT scans of the retina (location indicated by green arrow) taken 4 weeks before intravitreal injection of GSO30-DP. Bilateral hyporeflective cyst-like spaces (shown in light-blue circles) are commonly found in late-stage RP and represent outer retinal degeneration. 


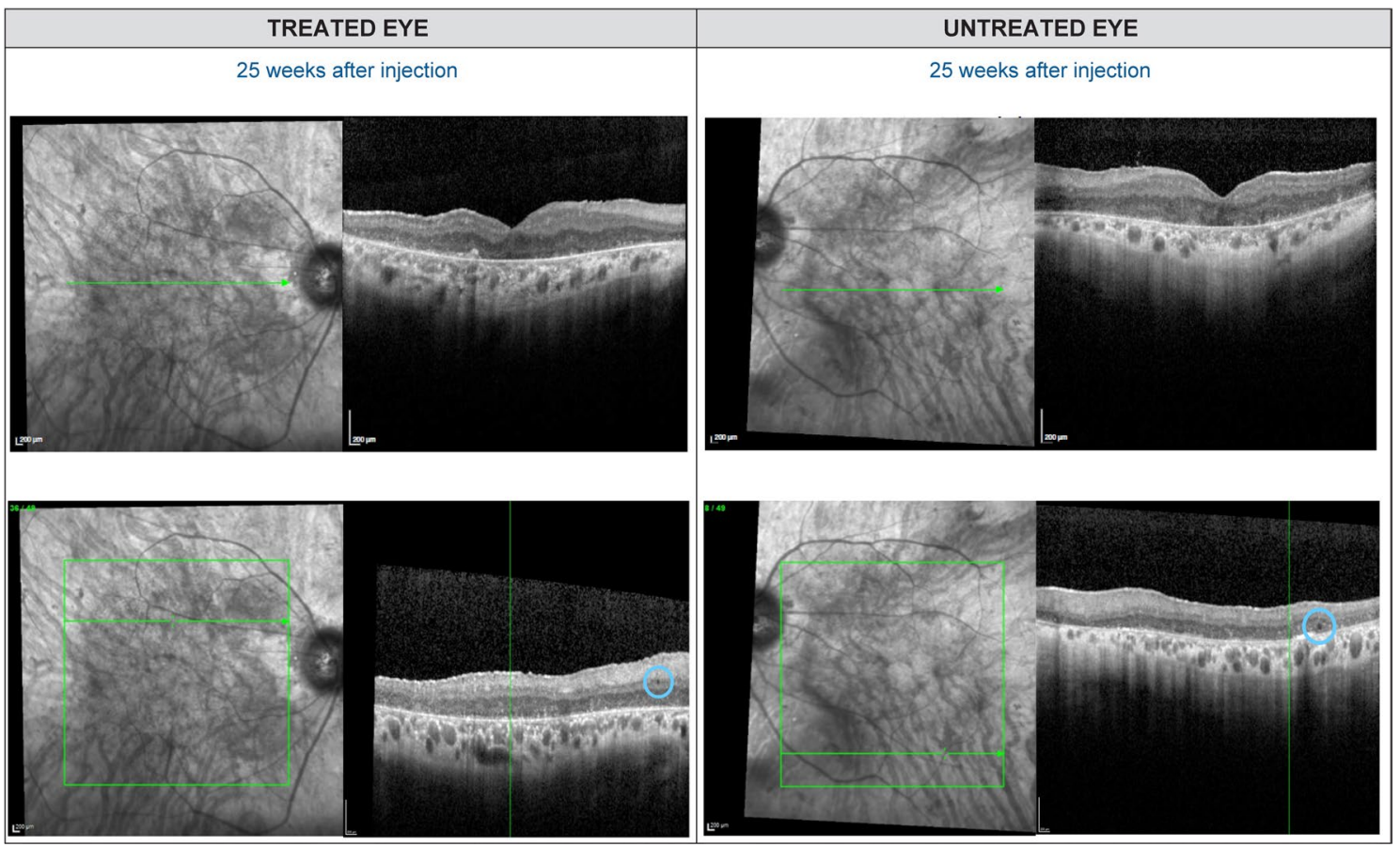

Extended Data Fig. 4 | OCT imaging of the retina 25 weeks after injection. OCT scans of the retina (location indicated by green arrow) taken 25 weeks after intravitreal injection of GS030-DP. Bilateral hyporeflective cyst-like spaces (shown in light-blue circles) are commonly found in late-stage RP and represent outer retinal degeneration. 


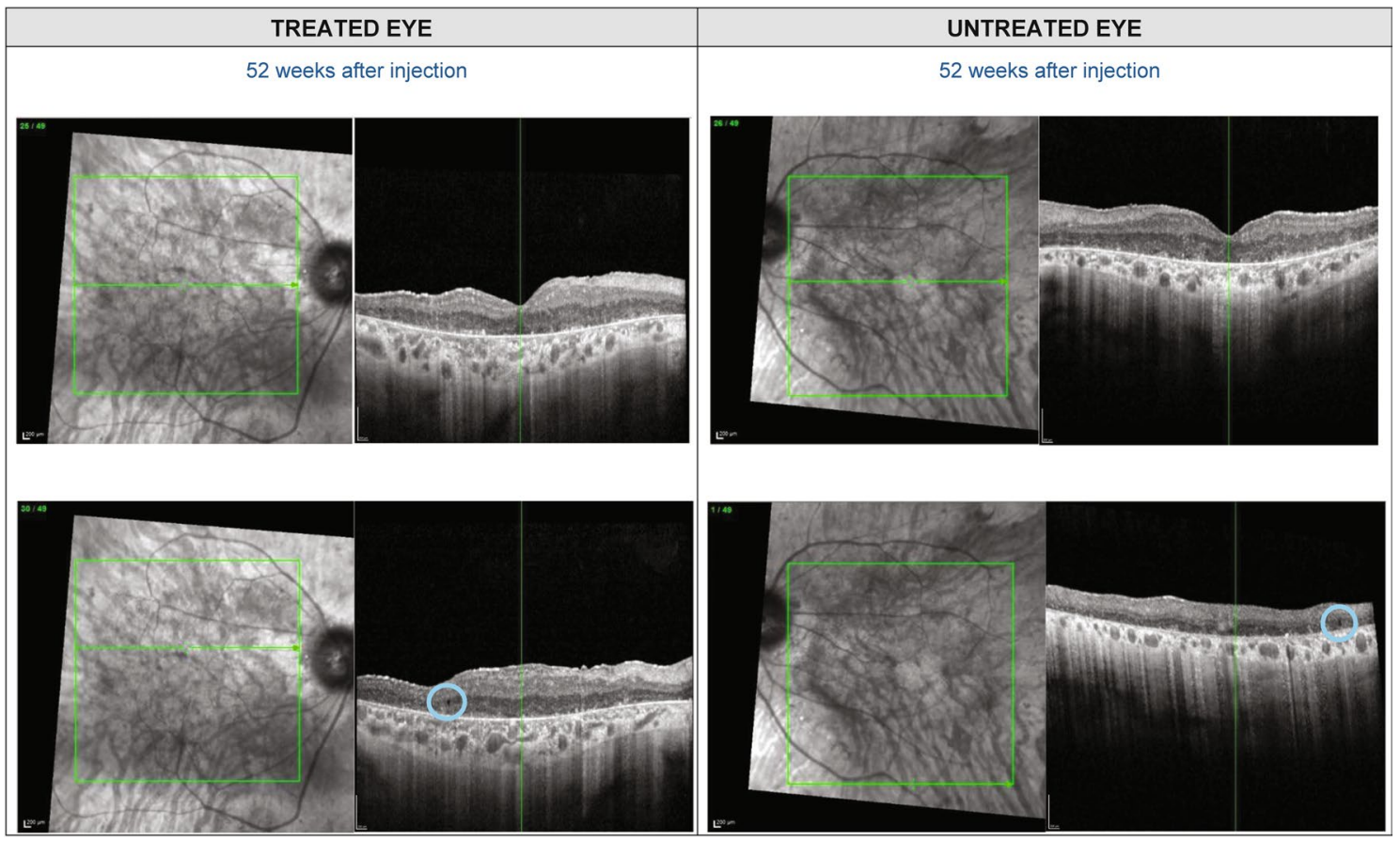

Extended Data Fig. 5 | OCT imaging of the retina 52 weeks after injection. OCT scans of the retina (location indicated by green arrow) taken 52 weeks after intravitreal injection of GS030-DP. Bilateral hyporeflective cyst-like spaces (shown in light-blue circles) are commonly found in late-stage RP and represent outer retinal degeneration. 


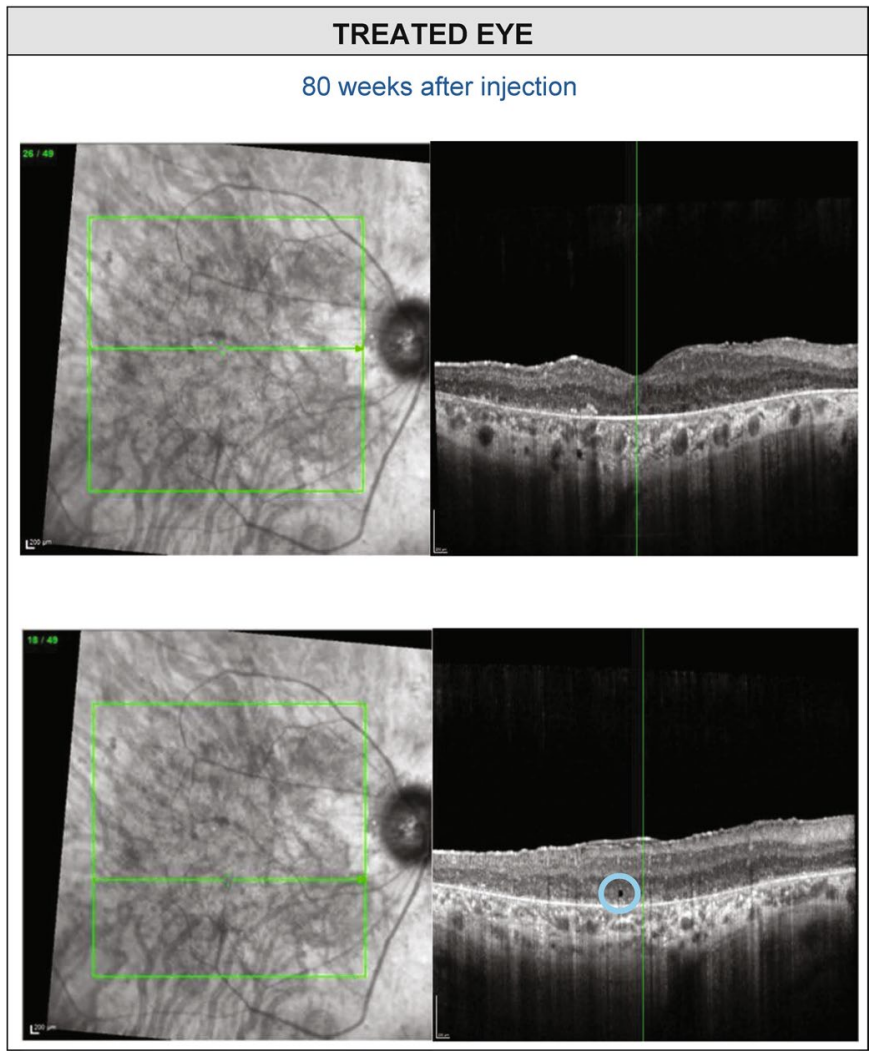

Extended Data Fig. 6 | OCT imaging of the retina 80 weeks after injection. OCT scans of the retina (location indicated by green arrow) taken 80 weeks after intravitreal injection of GS030-DP. Only the treated eye was assessed on that visit. Hyporeflective cyst-like spaces (example shown in light-blue circle) are commonly found in late-stage RP and represent outer retinal degeneration. 


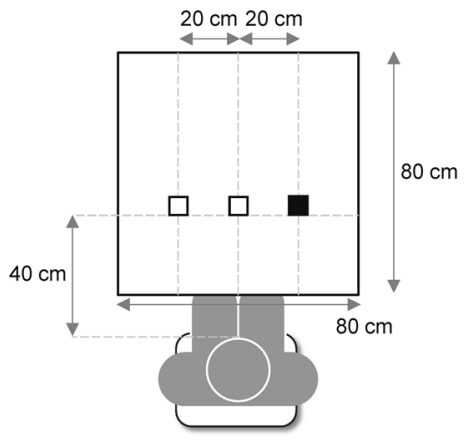

$\square$ Possible object positions

- Object (example)

$80 \mathrm{~cm}$

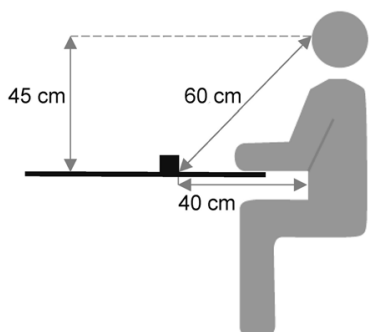

Extended Data Fig. 8 | Schematic of vision test 1. Test 1: perceiving, locating, and touching a single object. The subject had to perceive, locate, and touch a single object placed on a white table $(80 \mathrm{~cm} \times 80 \mathrm{~cm} ; 67.20 \times 50.90$ visual angle $)$ along an imaginary line at $40 \mathrm{~cm}$ from the subject $(60 \mathrm{~cm}$ from the eyes of the subject), and $20 \mathrm{~cm}$ to the right or to the left (18.40) or in front of the subject. The object was either a notebook $(12.5 \mathrm{~cm} \times 17.5 \mathrm{~cm} ; 10.80 \times 10.30)$ or a staple box $(3 \mathrm{~cm} \times 5.5 \mathrm{~cm} ; 2.80 \times 3.70)$, displayed individually in three different contrasts (Michelson contrasts $40 \%$, 55\%, and $100 \%$; notebook: RMS contrasts $0.41,0.53,0.80$; staple box: RMS contrasts $0.13,0.16,0.21)$ in a random order. 


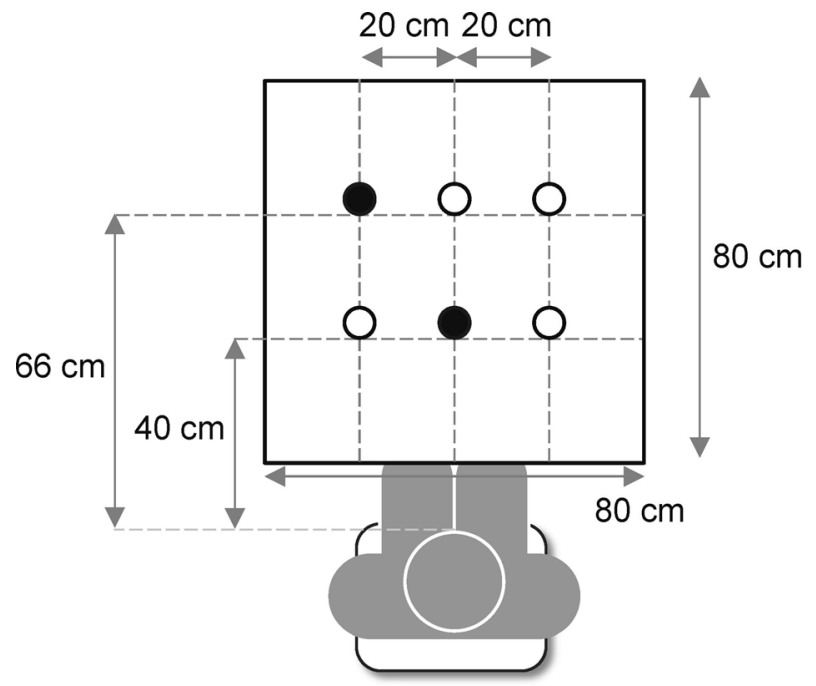

\section{Possible tumbler positions \\ - Tumbler (example)}

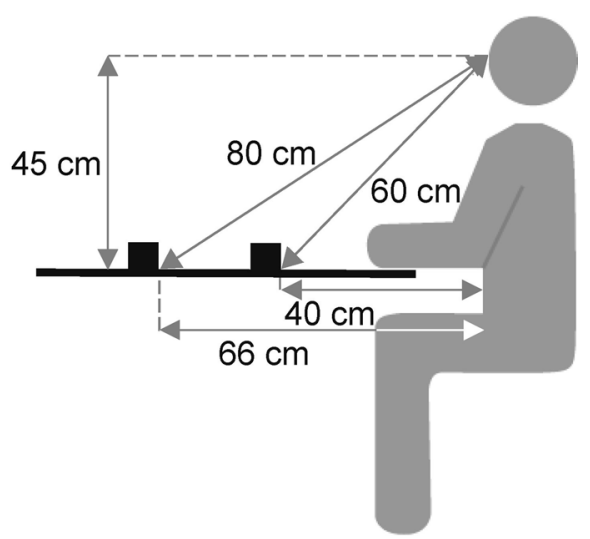

Extended Data Fig. 9 | Schematic of vision test 2. Test 2: perceiving, counting, and locating more than one object. The subject had to count and locate two or three tumblers placed on a white table $\left(80 \mathrm{~cm} \times 80 \mathrm{~cm} ; 67.2^{\circ} \times 50.9^{\circ}\right.$ visual angle) and to point at them without touching. Tumblers $(6 \mathrm{~cm}$ diameter and $6 \mathrm{~cm}$ height, $5.5^{\circ}$ and $8.1^{\circ}$ at $40 \mathrm{~cm}, 4.2^{\circ}$ and $5.8^{\circ}$ at $66 \mathrm{~cm}$ ) were positioned at two or three of six possible positions along two imaginary lines: at $40 \mathrm{~cm}$ from the subject $\left(60 \mathrm{~cm}\right.$ from the eyes of the subject), and $20 \mathrm{~cm}$ to the right or to the left $\left(18.4^{\circ}\right)$ or in front of the subject; or at $66 \mathrm{~cm}$ from the subject $\left(80 \mathrm{~cm}\right.$ from the eyes of the subject), and $20 \mathrm{~cm}$ to the right or to the left $\left(14^{\circ}\right)$ or in front of the subject. The objects were displayed in three different contrasts (Michelson contrasts $40 \%, 55 \%$, and 100\%; RMS contrasts $0.29,0.33,0.41$ ) in a random order. 


\section{Reporting Summary}

Nature Research wishes to improve the reproducibility of the work that we publish. This form provides structure for consistency and transparency in reporting. For further information on Nature Research policies, see our Editorial Policies and the Editorial Policy Checklist.

\section{Statistics}

For all statistical analyses, confirm that the following items are present in the figure legend, table legend, main text, or Methods section.

$\mathrm{n} / \mathrm{a}$ Confirmed

$\bigotimes$ The exact sample size $(n)$ for each experimental group/condition, given as a discrete number and unit of measurement

$\square$ \ A statement on whether measurements were taken from distinct samples or whether the same sample was measured repeatedly

The statistical test(s) used AND whether they are one- or two-sided

Only common tests should be described solely by name; describe more complex techniques in the Methods section.

Х $\square$ A description of all covariates tested

$\bigotimes$ A description of any assumptions or corrections, such as tests of normality and adjustment for multiple comparisons

$\triangle$ A full description of the statistical parameters including central tendency (e.g. means) or other basic estimates (e.g. regression coefficient) AND variation (e.g. standard deviation) or associated estimates of uncertainty (e.g. confidence intervals)

For null hypothesis testing, the test statistic (e.g. $F, t, r$ ) with confidence intervals, effect sizes, degrees of freedom and $P$ value noted Give $P$ values as exact values whenever suitable.

Х $\square$ For Bayesian analysis, information on the choice of priors and Markov chain Monte Carlo settings

Х $\square$ For hierarchical and complex designs, identification of the appropriate level for tests and full reporting of outcomes

$\triangle \square$ Estimates of effect sizes (e.g. Cohen's $d$, Pearson's $r$ ), indicating how they were calculated

\section{Our web collection on statistics for biologists contains articles on many of the points above.}

\section{Software and code}

Policy information about availability of computer code

Data collection All EEG data were collected by the EEGO v1.9.1 software and Arduino custom code for data acquisition (software Processing version 3.5.4). The code for EEG data processing and spectral analysis is available at the following URL: https://github.com/JBDSA/OptoRehabEEG

Data analysis Analyses of behavioral data were conducted in R, version 4.0.3.. EEG data were preprocessed and analyzed using the EEGLAB toolbox version 14.1.0 in MATLAB (versions R2019a \& R2020a), ICLabel version 1.2.6 using the default option (EEGLAB plug-in), and MATLAB custom code. Statistical analyses of EEG data were conducted in JASP, version 0.11.1.

For manuscripts utilizing custom algorithms or software that are central to the research but not yet described in published literature, software must be made available to editors and

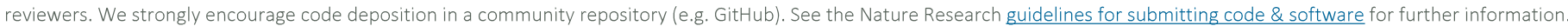

Policy information about availability of data

All manuscripts must include a data availability statement. This statement should provide the following information, where applicable:

- Accession codes, unique identifiers, or web links for publicly available datasets

- A list of figures that have associated raw data

- A description of any restrictions on data availability

All requests for raw and analyzed data are promptly reviewed by Gensight Biologics to verify if the request is subject to any intellectual property or confidentiality obligations. Patient-related data not included in the paper were generated as part of clinical trials and may be subject to patient confidentiality. Any data that can be shared will be released via a Material Transfer Agreement. All raw and analyzed image data can be found at:

https://passageinnovation-my.sharepoint.com/:f:/g/personal/mtaiel_gensight-biologics_com/EkUITiEa4AxNs_YryLq7fT8BGpdYkXZMWWtDK6Wg-fcQfA 
Please select the one below that is the best fit for your research. If you are not sure, read the appropriate sections before making your selection.

\ Life sciences

Behavioural \& social sciences

Ecological, evolutionary \& environmental sciences

For a reference copy of the document with all sections, see nature.com/documents/nr-reporting-summary-flat.pdf

\section{Life sciences study design}

All studies must disclose on these points even when the disclosure is negative.

Sample size $\quad$ No formal statistical hypothesis or sample size estimation were established for the PIONEER study. The sample size follows the traditional $3+$ 3 dose escalation design for the 3 dose levels (Vignal C et al. Safety of rAAV2/2-ND4 Gene Therapy for Leber Hereditary Optic Neuropathy. Ophthalmology. 2018 Jun;125(6):945-947. doi: 10.1016/j.ophtha.2017.12.036.). Sample size of PIONEER is consistent with previous early phase studies of gene therapy in orphan indications (Maguire AM et al. Age-dependent effects of RPE65 gene therapy for Leber's congenital amaurosis: a phase 1 dose-escalation trial. Lancet. 2009 Nov 7;374(9701):1597-605. doi: 10.1016/S0140-6736(09)61836-5).

Data exclusions No data were excluded from the analyses.

Replication The first visual test "finding notebook or staple box" included a total of 40 randomized notebook/staple box with three contrasts replicate trials distributed across three conditions: natural binocular, natural monocular, and stimulated monocular $(6,6$, and 28 trials, respectively). The second visual test "counting tumblers and locating them" test included a total of 25 randomized 2 objects/3objects with three contrasts replicate trials distributed across three conditions: natural binocular, natural monocular, and stimulated monocular $(3,3$, and 19 trials, respectively). The visual detection task coupled with EEG recordings included a total of 183 randomized object/no-object replicate trials distributed across three conditions: natural binocular, natural monocular, and stimulated monocular (60, 60, and 63 trials, respectively).

Randomization No randomization scheme was applied to treatment allocation. The patient's worse-seeing eye was selected for treatment with combined GS030 therapy.

Blinding

Not applicable, as this is an open-label study.

\section{Reporting for specific materials, systems and methods}

We require information from authors about some types of materials, experimental systems and methods used in many studies. Here, indicate whether each material, system or method listed is relevant to your study. If you are not sure if a list item applies to your research, read the appropriate section before selecting a response.

\begin{tabular}{l|l} 
Materials \& experimental systems \\
\hline$n / a$ & Involved in the study \\
\hline & $\square$ Antibodies \\
\hline & $\square$ Eukaryotic cell lines \\
$\square$ & $\square$ Palaeontology and archaeology \\
$\bigotimes$ & $\square$ Animals and other organisms \\
$\square$ & $\square$ Human research participants \\
$\square$ & $\bigotimes$ Clinical data \\
$\square$ & $\square$ Dual use research of concern
\end{tabular}

Methods

$\mathrm{n} / \mathrm{a}$ Involved in the study

$\triangle \square$ ChIP-seq

Х $\square$ Flow cytometry

Х $\square$ MRI-based neuroimaging

\section{Human research participants}

Policy information about studies involving human research participants

Population characteristics

Study participants have non-syndromic late-stage retinitis pigmentosa (RP), are between 18 and 75 years of age, mixed gender, and have at most Light Perception vision in their worse-seeing eye. The reported male patient is 58 years old and has two pathogenic alleles on the USH2A gene (NM_206933.2) that were identified by targeted next-generation sequencing (Audo et al., 2012) and segregation analysis using samples from his parents. The first pathogenic allele is on exon 13: c.2299del p.(Glu767Serfs*21) (Eudy et al., 1998), inherited from his father. The second pathogenic allele is a complex allele with exon 22 c.4714C>T p.Leu1572Phe (Song et al., 2011) and exon 50 c.9882C>G p.Cys3294Trp (Nishiguchi et al., 2013), inherited from his mother.

Recruitment

Participants in the PIONEER study are selected by study investigators according to the protocol selection criteria. They have to sign a detailed informed consent form before study enrollment, as per Good Clinical Practice and local regulations. The participation to the study was proposed to all patients who fulfilled the inclusion criteria across the three centers and there was no bias in the study recruitment. 

produits de santé (ANSM, France), the US Federal Drug and Food Administration (FDA, USA), the Medicines and Healthcare products Regulatory Agency (MRHA, UK); and the following national/local ethic committees and institutional review board: Comité de Protection des Personnes [CPP] Île-de-France III, France; North-East York Research Ethics Committee (REC), UK; Human Research Protection Office (HRPO) at the University of Pittsburg, Pittsburg, USA.

Note that full information on the approval of the study protocol must also be provided in the manuscript.

\section{Clinical data}

Policy information about clinical studies

All manuscripts should comply with the ICMJE guidelines for publication of clinical research and a completed CONSORT checklist must be included with all submissions.

Clinical trial registration

Study protocol

Data collection

Outcomes

\section{NCT03326336}

Full study protocol is available in appendix of the case report manuscript

The PIONEER study is carried out at three investigational sites: the Centre Hospitalier National d'Ophtalmologie des Quinze-Vingts (Paris, France), the Moorfields Eye Hospital NHS Foundation Trust (London, United Kingdom), and the Department of Ophthalmology at the University of Pittsburgh School of Medicine (Pittsburgh, USA). Patient recruitment started in September 2018 and is ongoing.

The primary objective of the PIONEER study is to evaluate the safety and tolerability of escalating doses of GSO30-DP administered via a single IVT injection and repeated light stimulation using the GSO3O-MD goggles, in subjects with non-syndromic RP.

The primary endpoint is assessed at Week 52/Year 1 based on local and systemic safety issues, specifically those related to IVT of GSO3O-DP and the subsequent repeated use of GSO3O-MD, as assessed by incidence of adverse events (AEs).

The secondary objectives of the PIONEER study, that have been reported, are to:

- Evaluate the treatment effect of GSO3O combined therapy, as assessed by vision and ocular measures, including visual acuity and visual function.

- Compare visual acuity and visual function before and after gene transfer, with and without GSO30-MD.

The reported secondary endpoints are:

- Assessment of the treatment effect on visual function and functional vision, with the change from baseline to Week 52 of parameters, including visual perception tests, eye-hand coordination and visual exploration.

-Assessment of the treatment effect on structural changes of the posterior pole of the fundus from baseline to Week 52 with parameters measured with SD-OCT, color fundus photography, and fundus auto fluorescence (FAF). 